\title{
Albany/FELIX: a parallel, scalable and robust, finite element, first-order Stokes approximation ice sheet solver built for advanced analysis
}

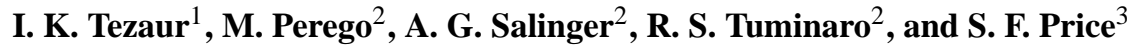 \\ ${ }^{1}$ Quantitative Modeling and Analysis Department, Sandia National Laboratories, \\ P.O. Box 969, MS 9159, Livermore, CA 94551, USA \\ ${ }^{2}$ Computational Mathematics Department, Sandia National Laboratories, P.O. Box 5800, \\ MS 1320, Albuquerque, NM 87185, USA \\ ${ }^{3}$ Fluid Dynamics and Solid Mechanics Group, Los Alamos National Laboratory, \\ P.O. Box 1663, MS B216, Los Alamos, NM 87545, USA
}

Correspondence to: I. K. Tezaur (ikalash@sandia.gov)

Received: 30 September 2014 - Published in Geosci. Model Dev. Discuss.: 24 November 2014

Revised: 18 March 2015 - Accepted: 2 April 2015 - Published: 27 April 2015

\begin{abstract}
This paper describes a new parallel, scalable and robust finite element based solver for the first-order Stokes momentum balance equations for ice flow. The solver, known as Albany/FELIX, is constructed using the component-based approach to building application codes, in which mature, modular libraries developed as a part of the Trilinos project are combined using abstract interfaces and template-based generic programming, resulting in a final code with access to dozens of algorithmic and advanced analysis capabilities. Following an overview of the relevant partial differential equations and boundary conditions, the numerical methods chosen to discretize the ice flow equations are described, along with their implementation. The results of several verification studies of the model accuracy are presented using (1) new test cases for simplified two-dimensional (2-D) versions of the governing equations derived using the method of manufactured solutions, and (2) canonical ice sheet modeling benchmarks. Model accuracy and convergence with respect to mesh resolution are then studied on problems involving a realistic Greenland ice sheet geometry discretized using hexahedral and tetrahedral meshes. Also explored as a part of this study is the effect of vertical mesh resolution on the solution accuracy and solver performance. The robustness and scalability of our solver on these problems is demonstrated. Lastly, we show that good scalability can be achieved by preconditioning the iterative linear solver using a new algebraic
\end{abstract}

multilevel preconditioner, constructed based on the idea of semi-coarsening.

\section{Introduction}

In its fourth assessment report (AR4), the Intergovernmental Panel on Climate Change (IPCC) declined to include estimates of future sea-level rise from ice sheet dynamics due to the inability of ice sheet models to mimic or explain observed dynamic behaviors, such as the acceleration and thinning then occurring on several of Greenland's large outlet glaciers (IPCC, 2007). Since the AR4, increased support from United States, United Kingdom, and European Union funding agencies has enabled concerted efforts towards improving the representation of ice dynamics in ice sheet models and towards their coupling to other components of Earth system models (ESMs) (Little, 2007; Lipscomb et al., 2008; van der Veen et al., 2010). Thanks to this support, there has recently been tremendous progress in the development of "next generation" community-supported ice sheet models (e.g., Bueler and Brown, 2009; Rutt et al., 2009; Larour et al., 2012; Gagliardini et al., 2013; Brinkerhoff and Johnson, 2013; Lipscomb et al., 2013) able to perform realistic, highresolution, continental-scale simulations. These models run on massively parallel high-performance computing (HPC) 
architectures using $10^{2}-10^{4}$ processes and employ modern, well-supported solver libraries (e.g., PETSC, Balay et al., 2008, and Trilinos, Heroux et al., 2005). A primary development focus has been on improving the representation of the momentum balance equations over the "shallow ice" (SIA; Hutter, 1983) and "shallow-shelf" (SSA; Morland, 1987) approximations through the inclusion of both vertical shear and membrane stresses over the entire model domain (e.g., Pattyn, 2002). These approaches include "hybrid" models (a combination of SIA and SSA; Bueler and Brown, 2009; Pollard and Deconto, 2009; Goldberg and Sergienko, 2011), socalled "higher-order" models (Pattyn, 2003), "full" Stokes models (Larour et al., 2012; Leng et al., 2012; Gagliardini et al., 2013), and combinations of a range of approximations up to and including full Stokes (Seroussi et al., 2012). By accounting for both vertical and horizontal stress gradients, the aforementioned models allow for more realistic and accurate simulations of outlet glaciers, ice streams, and ice shelves, as well as modeling of the transfer of perturbations from marginal to inland regions.

Other significant improvements in ice sheet modeling frameworks include the integration of unstructured (Larour et al., 2012; Gagliardini et al., 2013; Brinkerhoff and Johnson, 2013) or adaptive (Cornford et al., 2013) meshes, which allows the focusing of resolution and computational power in regions of dynamic complexity. Also becoming standard is the use of formal optimization and data assimilation techniques for generating realistic model initial conditions. Surface observations are used to infer poorly known ice properties or parameters, such as the friction coefficient at the ice-bedrock interface (e.g., Morlighem et al., 2010; Larour et al., 2012; Gillet-Chaulet et al., 2012; Brinkerhoff and Johnson, 2013) or the rheology of floating ice shelves (Khazendar et al., 2009), allowing for a quantifiably "optimal" match between modeled and observed velocities. Recently, these approaches have been extended to simultaneously optimize both model parameter fields and uncertain initial condition fields, while also accounting for forcing from climate models in order to minimize transient shocks when coupling to climate forcing (Perego et al., 2014). Other recent and noteworthy optimization improvements include the assimilation of time-dependent observations (e.g., Goldberg and Heimbach, 2013) and the estimation of formal uncertainties for optimized parameter fields (Petra et al., 2015).

The latter capability - the characterization of parameter uncertainties - represents a critical first step towards formal uncertainty quantification (UQ) of ice sheet model output quantities of interest, such as estimates of future sea-level rise. For this process to be computationally tractable during both the inverse (parameter estimation and uncertainty assignment) and forward propagation steps, it is crucial to have robust, efficient, and scalable solves on HPC platforms (Isaac et al., 2014). This, in turn, requires advanced dynamical core capabilities, such as access to model derivatives (e.g., the Jacobian matrix), and advanced algorithms for the solution of the nonlinear and linear equations. These same requirements of robustness, efficiency, and scalability hold for the inclusion of ice sheet models as fully coupled components of large-scale, high-resolution ESMs.

In this paper, we introduce and focus on a new momentum balance solver for land ice simulations based on the firstorder approximation of the nonlinear Stokes flow model for glaciers and ice sheets. This new solver, Albany/FELIX (Finite Elements for Land Ice eXperiments, described in more detail below), either already includes many of the capabilities discussed above or is designed to allow for their easy implementation at later stages of development. Here, we present algorithms and software that lead to a robust nonlinear solution procedure (including the use of automatic differentiation (AD) technologies), scalable linear algebra, and the ability to use unstructured and highly refined grids.

The remainder of this paper is organized as follows. In Sect. 2, we describe in detail our mathematical model for glaciers and ice sheets, giving the relevant assumptions, partial differential equations, boundary conditions, and parameter values. Our numerical methods for discretizing this model and their implementation in Albany/FELIX are summarized in Sect. 3. In Sect. 4, which focuses on verification of the Albany/FELIX code using the method of manufactured solutions, two new test cases are derived for simplified twodimensional (2-D) versions of the first-order Stokes equations and used in a convergence verification study involving several types and orders of finite elements. In Sect. 5, further verification of the accuracy of solutions computed with our solver is performed using canonical ice sheet modeling test cases. The results of a mesh convergence study on a realistic Greenland ice sheet geometry are then discussed in Sect. 6 . This study provides insight into the effects of the parallel domain decomposition on solver convergence, and the effect of the vertical mesh resolution on solution accuracy. We then describe our robust, nonlinear solver, which uses homotopy continuation with respect to the regularization parameter in the calculation of the ice effective viscosity. The solver's robustness and scalability is demonstrated on various Greenland ice sheet geometries, discretized using tetrahedral and hexahedral meshes. Finally, we show that improved scalability of our code can be achieved by preconditioning the iterative linear solver using an algebraic multilevel preconditioner, constructed based on the idea of semi-coarsening. A concluding summary is offered in Sect. 7. Here, we also touch briefly on the larger ice sheet modeling frameworks that Albany/FELIX is being incorporated into for treating the conservation of mass and energy and for performing prognostic runs in both standalone mode and as coupled components of ESMs.

One objective of this paper is to introduce a new parallel, scalable and robust finite element first-order Stokes solver for ice flow, namely Albany/FELIX, to the land ice and climate modeling communities. The article also contains several new 
contributions to the field of ice sheet modeling, which are most notably:

- the derivation of several new test cases based on the method of manufactured solutions for simplified 2-D forms of the first-order Stokes equations, which can be used to verify convergence to an exact solution for parts of the governing PDEs in any ice sheet code that discretizes these equations.

- the description of a homotopy continuation algorithm with respect to a regularization parameter in the ice effective viscosity expression, which greatly improves the robustness of a Newton nonlinear solver, especially in the absence of a good initial guess.

- insights into the effects of the parallel decomposition and vertical mesh spacing on solver performance and solution accuracy for ice sheet simulations.

- a new algebraic multilevel preconditioner, constructed based on the idea of semi-coarsening and ideal for meshes structured in the vertical direction, that delivers a scalable linear solve when combined with a preconditioned iterative method.

\section{First-order Stokes approximation mathematical model}

We consider a power-law viscous, incompressible fluid in a low Reynolds number flow, described by the first-order approximation to the nonlinear Stokes flow equations for glaciers and ice sheets (Dukowicz et al., 2010; Schoof and Hindmarsh, 2010). The first-order (FO) approximation, also referred to as the Blatter-Pattyn model (Pattyn, 2003; Blatter, 1995), follows from assumptions of a small geometric aspect ratio, $\delta=H / L$ (where $H$ and $L$ are characteristic length scales for the vertical and horizontal dimensions, respectively, and $H \ll L$ ), and the assumption that the normal vectors to the ice sheet's upper and lower surfaces, $\boldsymbol{n} \in \mathbb{R}^{3}$, are nearly vertical:

$\boldsymbol{n}^{T} \approx\left(\mathcal{O}(\delta), \mathcal{O}(\delta), \pm 1+\mathcal{O}\left(\delta^{2}\right)\right)$.

Effectively, the FO approximation is derived by neglecting $\mathcal{O}\left(\delta^{2}\right)$ terms in the Stokes equations and respective boundary conditions (discussed in more detail in Appendix A). Numerical discretization of the FO Stokes equations gives rise to a much smaller discrete system than numerical discretization of the full Stokes equations. Moreover, discretization of the FO Stokes system gives rise to a "nice" elliptic coercive problem, in contrast to the notoriously difficult saddle-point problem obtained when discretizing the full Stokes system.

Let $u$ and $v$ denote the $x$ and $y$ components of the ice velocity vector $\boldsymbol{u} \equiv(u, v)^{T} \in \mathbb{R}^{2}$, respectively. The FO approx- imation consists of the following system of partial differential equations (PDEs):

$$
\left\{\begin{array}{l}
-\nabla \cdot\left(2 \mu \dot{\boldsymbol{\epsilon}}_{1}\right)+\rho g \frac{\partial s}{\partial x}=0, \\
-\nabla \cdot\left(2 \mu \dot{\boldsymbol{\epsilon}}_{2}\right)+\rho g \frac{\partial s}{\partial y}=0
\end{array}\right.
$$

where $g$ denotes the gravitational acceleration, $\rho$ denotes the ice density, and $s \equiv s(x, y)$ denotes the upper surface boundary:

$\Gamma_{s} \equiv\left\{(x, y, z) \in \mathbb{R}^{3} \mid z=s(x, y)\right\}$.

In the most general, three-dimensional (3-D) case of the FO approximation, the strain-rate tensor

$\dot{\boldsymbol{\epsilon}} \equiv\left(\dot{\boldsymbol{\epsilon}}_{1}, \dot{\boldsymbol{\epsilon}}_{2}\right) \in \mathbb{R}^{3 \times 2}$

is given by the following components

$\dot{\boldsymbol{\epsilon}}_{1}^{T}=\left(2 \dot{\epsilon}_{x x}+\dot{\epsilon}_{y y}, \dot{\epsilon}_{x y}, \dot{\epsilon}_{x z}\right) \in \mathbb{R}^{3}$,

and

$\dot{\boldsymbol{\epsilon}}_{2}^{T}=\left(\dot{\epsilon}_{x y}, \dot{\epsilon}_{x x}+2 \dot{\epsilon}_{y y}, \dot{\epsilon}_{y z}\right) \in \mathbb{R}^{3}$,

where

$\dot{\epsilon}_{x x}=\frac{\partial u}{\partial x}, \quad \dot{\epsilon}_{y y}=\frac{\partial v}{\partial y}, \quad \dot{\epsilon}_{x y}=\frac{1}{2}\left(\frac{\partial u}{\partial y}+\frac{\partial v}{\partial x}\right)$,

$\dot{\epsilon}_{x z}=\frac{1}{2} \frac{\partial u}{\partial z}, \quad \dot{\epsilon}_{y z}=\frac{1}{2} \frac{\partial v}{\partial z}$.

The effective viscosity $\mu$ can be derived using Glen's flow law (Cuffey and Paterson, 2010; Nye, 1957) as

$\mu=\frac{1}{2} A^{-\frac{1}{n}} \dot{\epsilon}_{\mathrm{e}}^{\frac{1}{n}-1}$,

where $\dot{\epsilon}_{\mathrm{e}}$ is the effective strain rate, given by

$\dot{\epsilon}_{\mathrm{e}}^{2} \equiv \dot{\epsilon}_{x x}^{2}+\dot{\epsilon}_{y y}^{2}+\dot{\epsilon}_{x x} \dot{\epsilon}_{y y}+\dot{\epsilon}_{x y}^{2}+\dot{\epsilon}_{x z}^{2}+\dot{\epsilon}_{y z}^{2}$.

In Eq. (8), $A$ is the flow rate factor and $n$ is the Glen's (power) law exponent, typically taken equal to 3 for ice sheets. Hence, $\mu$ Eq. (8) is a nonlinear expression, and the system Eq. (2) is a nonlinear, elliptic system of PDEs. The flow-law rate factor $A$ is strongly temperature dependent, and can be described through the Arrhenius relation,

$A(T)=A_{0} \exp \left(-\frac{Q}{R T^{*}}\right)$,

where $A_{0}$ denotes a constant of proportionality, $Q$ denotes the activation energy for ice creep, $T^{*}$ denotes the ice temperature in Kelvin $(\mathrm{K})$ corrected for the pressure melting point dependence, and $R$ denotes the universal gas constant. For more details involving the relation between the flow factor 
and temperature Eq. (10), the reader is referred to Cuffey and Paterson (2010). For completeness, the expressions for the Cauchy stress tensor $\sigma$ and the pressure $p$ in the FO approximation are provided:

$\boldsymbol{\sigma}=2 \mu\left(\dot{\boldsymbol{\epsilon}}_{1}, \dot{\boldsymbol{\epsilon}}_{2}, \mathbf{0}\right)^{T}-\rho g(s-z) \mathbf{I}$,

$p=\rho g(s-z)-2 \mu\left(\dot{\epsilon}_{x x}+\dot{\epsilon}_{y y}\right)$,

where $\mathbf{0}=(0,0,0)^{T}$ and $\mathbf{I}$ is the $3 \times 3$ identity tensor. The equations Eq. (2) are specified on a bounded 3-D domain, denoted by $\Omega$, with boundary

$\Gamma \equiv \Gamma_{s} \cup \Gamma_{b} \cup \Gamma_{1}$

Here, $\Gamma_{s}$ is the upper surface boundary Eq. (3), and

$\Gamma_{b}=\left\{(x, y, z) \in \mathbb{R}^{3} \mid z=b(x, y)\right\}$,

$\Gamma_{1}=\left\{(x, y, z) \in \mathbb{R}^{3} \mid l(x, y)=0\right\}$,

are the lower and (vertical) lateral surface boundaries, respectively. The relevant boundary conditions on $\Gamma$ are

(a) a stress-free (homogeneous Neumann) boundary condition on the upper surface boundary

$\dot{\boldsymbol{\epsilon}}_{1} \cdot \boldsymbol{n}=\dot{\boldsymbol{\epsilon}}_{2} \cdot \boldsymbol{n}=0, \quad$ on $\Gamma_{s}$.

(b) either a no-slip or a sliding boundary condition on the lower surface:

$\left\{\begin{array}{l}u=v=0, \text { on } \Gamma_{0} \\ 2 \mu \dot{\boldsymbol{\epsilon}}_{1} \cdot \boldsymbol{n}+\beta u=0,2 \mu \dot{\boldsymbol{\epsilon}}_{2} \cdot \boldsymbol{n}+\beta v=0, \text { on } \Gamma_{\beta},\end{array}\right.$

where $\Gamma_{b}$ is partitioned as $\Gamma_{b}=\Gamma_{0} \cup \Gamma_{\beta}$ with $\Gamma_{0} \cap \Gamma_{\beta}=$ $\varnothing$, and $\beta \equiv \beta(x, y) \geq 0$ is the basal sliding coefficient. Note that we assume that the partitioning of $\Gamma_{b}$ is known a priori. In practice, this would be specified (through a conservation of energy equation) by locating regions of the bed for which the temperature is at the pressure melting point. It is often more practical to enforce a quasi-no-slip Robin boundary condition on $\Gamma_{0}$ by setting $\beta$ to a large value and always using the equation on the second line of Eq. (16) (e.g., $\beta=10^{7} \mathrm{kPa} \mathrm{a} \mathrm{m}^{-1}$ ).

(c) On the lateral boundaries, one of two boundary conditions is applied: either a kinematic (Dirichlet) boundary condition

$u=u_{1}, \quad v=v_{1}$, on $\Gamma_{1}$, where $u_{1}$ and $v_{1}$ are prescribed values of the ice velocities on the lateral boundary, or a dynamic (Neumann) boundary condition

$2 \mu \dot{\boldsymbol{\epsilon}}_{i} \cdot \boldsymbol{n}-\rho g(s-z) \boldsymbol{n}=\rho_{\mathrm{w}} g \min (z, 0) \boldsymbol{n}$, on $\Gamma_{1}$,

for $i=1,2$, where $\rho_{\mathrm{w}}$ denotes the density of water. In Eq. (18), it has been assumed that the coordinate system has been oriented such that $z$ is strictly elevation (that is, $z=0$ at sea level and values of $z$ increase for higher elevations) (MacAyeal et al., 1996). The boundary condition Eq. (18) is derived by assuming that the ice shelf is in hydrostatic equilibrium with the air/water that surrounds it and is often referred to as an "open-ocean" boundary condition, as it takes into account the pressure exerted on the ice shelf by the neighboring ocean. For some canonical benchmark experiments performed here (see Sect. 5.1), periodic lateral boundary conditions are prescribed as well.

The values of the parameters that appear in the first-order Stokes equations and the boundary conditions described above and used herein are summarized in Table 1. From this point forward, the new first-order Stokes approximation momentum balance solver will be referred to as " $\mathrm{Al}$ bany/FELIX'. In this code, the numerical discretization of Eq. (2) uses Trilinos, a suite of modular software libraries (described in detail in Heroux et al., 2005).

\section{Numerical discretization and implementation}

The model described in Sect. 2 is discretized and solved using a collection of algorithms and software implementations that were selected for accuracy, flexibility, robustness, and scalability. The following brief discussion of the methods presumes prior knowledge of Galerkin finite element approaches and Newton-Krylov based nonlinear solvers (Strang and Fix, 1973; Pawlowski et al., 2006).

\subsection{Numerical methods}

The PDEs for the FO Stokes model defined by Eq. (2) and the associated boundary conditions are discretized using the classical Galerkin finite element method (FEM) (Hughes, 2000).

Let $\mathcal{V}$ denote the Hilbert space given by

$\mathcal{V} \equiv \mathcal{V}(\Omega)=\left\{\phi \in H^{1}(\Omega):\left.\phi\right|_{\Gamma_{0}}=0\right\}$

where $H^{1}(\Omega)$ denotes the space of square-integrable functions whose first derivatives are also square integrable. Following the classical Galerkin FEM methodology, the weak form of the problem is obtained by projecting each of the equations in Eq. (2) onto a test function in $\mathcal{V}$ Eq. (19) in the 
Table 1. Physical parameter values for first-order Stokes equations and boundary conditions*.

\begin{tabular}{|c|c|c|c|}
\hline Name & Value & Units & Description \\
\hline$A$ & $10^{-4}$ & $k^{-(n+1)} \mathrm{Pa}^{-n} \mathrm{a}^{-1}$ & Flow rate factor \\
\hline$n$ & 3 & - & Glen's flow law exponent \\
\hline$g$ & 9.8 & $\mathrm{~ms}^{-2}$ & Gravitational constant \\
\hline$\rho$ & 910 & $\mathrm{~kg} \mathrm{~m}^{-3}$ & Ice density \\
\hline$\rho_{\mathrm{W}}$ & 1025 & $\mathrm{~kg} \mathrm{~m}^{-3}$ & Ocean water density \\
\hline$R$ & 8.314 & $\mathrm{~J} \mathrm{~K}^{-1} \mathrm{~mol}^{-1}$ & Universal gas constant \\
\hline$A_{0}$ & $\begin{cases}1.30 \times 10^{7}, & \text { if } T<263 \mathrm{~K} \\
6.22 \times 10^{22}, & \text { if } T \geq 263 \mathrm{~K}\end{cases}$ & $k^{-(n+1)} \mathrm{Pa}^{-n} \mathrm{~s}^{-1}$ & Arrhenius constant of proportionality \\
\hline$Q$ & $\begin{array}{ll}6.00 \times 10^{4}, & \text { if } T<263 \mathrm{~K}, \\
1.39 \times 10^{5}, & \text { if } T \geq 263 \mathrm{~K},\end{array}$ & $\mathrm{~J} \mathrm{~mol}^{-1}$ & Activation energy for ice creep \\
\hline
\end{tabular}

*The symbol $k$ in the table denotes $\mathrm{km} \mathrm{m}^{1}$, i.e., $k=\mathrm{km} \mathrm{m}^{-1}=10^{3}$.

continuous $L^{2}$ inner product and integrating the second-order terms by parts. Toward this effect, the weak formulation of Eq. (2), for grounded ice, reads: find $u, v \in \mathcal{V}$ such that

$$
\left\{\begin{aligned}
& \int_{\Omega} 2 \mu \dot{\boldsymbol{\epsilon}}_{1}(u, v) \nabla \phi_{1} d \Omega+\int_{\Gamma_{\beta}} \beta u \phi_{1} d \Gamma \\
&+\int_{\Omega} \rho g \frac{\partial s}{\partial x} \phi_{1} d \Omega=0, \\
& \int_{\Omega} 2 \mu \dot{\boldsymbol{\epsilon}}_{2}(u, v) \cdot \nabla \phi_{2} d \Omega+\int_{\Gamma_{\beta}} \beta v \phi_{2} d \Gamma \\
&+\int_{\Omega} \rho g \frac{\partial s}{\partial y} \phi_{2} d \Omega=0,
\end{aligned}\right.
$$

for all $\phi_{1}, \phi_{2} \in \mathcal{V}(\Omega)$. The surface integral along the boundary appearing in Eq. (20) arises from integration by parts of the stress term in the variational form of the PDEs. This approach leads to a weak enforcement of the basal surface boundary condition Eq. (16) for the tangential stress, and straightforward implementation of the basal boundary conditions as an integrated boundary condition. (We believe, but have not rigorously shown, that the Gelerkin finite element approach for implementing the basal surface boundary condition enables one to circumvent robustness issues stemming from the discretization that were previously seen in our work with a finite difference discretization (Lemieux et al., 2011).)

Letting $\mathcal{F}\left(u, v ; \phi_{1}, \phi_{2}\right)$ denote the operator defining the left-hand side of Eq. (20), the problem defined by Eq. (20) is equivalent to finding the roots $u, v \in \mathcal{V}$ of the following nonlinear equation:

$\mathcal{F}\left(u, v ; \phi_{1}, \phi_{2}\right)=0, \quad \forall \phi_{1}, \phi_{2} \in \mathcal{V}$.

Equation (21) is an infinite-dimensional problem; a finitedimensional analog of Eq. (21) is obtained by replacing the infinite-dimensional space $\mathcal{V}$ by a finite-dimensional finite element space, $\mathcal{V}_{h}$, where $h$ is a length scale associated with a triangulation of the domain $\Omega$ into a set of disjoint finite elements $\Omega_{\mathrm{e}}\left(\Omega=\cup_{e=1}^{n_{\mathrm{el}}} \Omega_{\mathrm{e}}\right.$, where $n_{\mathrm{el}} \in \mathbb{N}$ is the number of finite elements in the triangulation).

Our implementation (a detailed discussion of which is given in Sect. 3.2) allows for tetrahedral (with either trilinear or triquadratic basis functions) or hexahedral elements (with bilinear or biquadratic basis functions) for 3-D problems. One reason a finite element approach was selected was for its flexibility in using unstructured grids with non-uniform mesh density to increase the resolution in areas of large velocity gradients, such as in the vicinity of outlet glaciers, while retaining relatively coarse meshes in the more static interior regions. In this paper, we present results on three different types of grids:

(i) structured uniform hexahedral grids,

(ii) unstructured uniform tetrahedral grids, and

(iii) unstructured non-uniform tetrahedral grids.

The structured hexahedral meshes are generated by creating a uniform quadrilateral grid of a 2-D horizontal cross section of a geometry $\Omega$, and extruding it in a uniform fashion as hexahedra in the vertical direction. Similarly, the uniform tetrahedral meshes are created by meshing a 2-D horizontal cross section of $\Omega$ using a uniform triangular mesh, extruding it in the vertical direction as prisms, then splitting each prism into three tetrahedra (Fig. 14) ${ }^{1}$. For the unstructured tetrahedral grids, an unstructured Delaunay triangle mesh of a 2-D cross section of $\Omega$ is generated based on some kind of refinement criteria (e.g., a static refinement based on the gradient of the velocity) using a meshing software (e.g., Triangle, a Delaunay triangulation mesh, Shewchuk, 1996), and extruded in the vertical direction in the same way as a structured triangular grid. More details on these meshes are provided in Sects. 5 and 6. Note that although all the meshes

\footnotetext{
${ }^{1}$ Another possibility, which we have not fully explored yet, is to use wedge elements on prisms.
} 
employed for the ice sheet application considered here were extruded (structured) in the vertical direction, our code base allows for completely unstructured grids.

A domain decomposition approach is used to compute the solution to the discretized nonlinear problem on distributed memory parallel computers. As a pre-processing step, the elements of the mesh are partitioned into one contiguous domain per processor to provide nearly equal work per processor. To do the partitioning, we used the decomposition utility (called decomp) available as a part of the Sandia Engineering Analysis Code Access System (SEACAS) database of Trilinos to create a linear decomposition of the 2-D mesh. Additional discussion of the parallel decompositions employed can be found in Sect. 6 .

The result of the discretization process is a large, sparse system of nonlinear algebraic equations for the two components of horizontal velocity at the nodes of the mesh (the discrete counterpart of Eq. 21). Our approach to solving this fully coupled, nonlinear system is Newton's method. An analytic Jacobian matrix is computed at each iteration of Newton's method using automatic differentiation (AD). The integration of AD into the Albany code base, both for Jacobians and for parameter derivatives for sensitivity analysis and UQ, has been a significant advantage of developing a new model in this framework. The matrix is stored in sparse form, with rows of the matrix distributed across the processors of the machine.

The resulting linear system is solved using a preconditioned iterative method. For the largest problems, we use multilevel preconditioning (described in Sect. 3.1.2) to achieve scalability, while incomplete LU (ILU) additive Schwarz preconditioners work well for modest problem sizes and processor counts. Since the model is symmetric, the conjugate gradient (CG) iterative linear solver is employed.

Because of the singularity in the viscosity formulation for stress-free solutions, such as when computing the nonlinear solution from a trivial initial guess, the Newton iteration does not reliably converge. To achieve a robust nonlinear solution procedure, we formulated and implemented a homotopy continuation approach that steps to the final solution by solving a series of nonlinear problems that reliably converge. The details of this algorithm are given in Sect. 3.1.1.

\subsubsection{Homotopy continuation algorithm}

Although the stress tensor $\sigma$ Eq. (11) is well defined for any differentiable function $\boldsymbol{u}$, the Glen's law effective viscosity Eq. (8) is not defined when $\boldsymbol{u}$ is a rigid movement or exactly 0 (because $n$ is typically taken to be greater than 1 ; see, e.g., Schoof, 2010; Chen et al., 2013). This can pose a problem for nonlinear solvers as the initial guess for $\boldsymbol{u}$ is often taken as uniform or 0 . To circumvent this difficulty, a regularization parameter $\gamma>0, \gamma \ll 1$ is added to the sum of the strain rates in the effective strain rate term of the effective viscosity
Eq. (8), yielding what we refer to as $\mu_{\gamma}$ :

$\mu_{\gamma}=\frac{1}{2} A^{-\frac{1}{n}}\left(\dot{\epsilon}_{\mathrm{e}}^{2}+\gamma\right)^{\left(\frac{1}{2 n}-\frac{1}{2}\right)}$, where $\lim _{\gamma \rightarrow 0} \mu_{\gamma}=\mu$.

One common practice is to define $\mu=\mu_{\gamma}$ in Eq. (8) using some small, fixed value for $\gamma$, e.g., $\gamma=10^{-10}$. Here, noting that the nonlinear solver often struggles to converge initially when using Newton's method, we use a variable $\gamma$ as the continuation parameter in a homotopy method (Algorithm 1). In this approach, a sequence of problems Eq. (2) is solved for a sequence of effective viscosities $\left\{\mu_{\gamma_{i}}\right\}$ for $i=1,2, \ldots$, with $0<\gamma_{i+1}<\gamma_{i}$, until $\gamma$ reaches its target value. We use a natural continuation procedure, where the final solution at one value of the continuation parameter $\alpha$ (defined in Algorithm 1) is used as the initial guess for the subsequent nonlinear problem. The continuation algorithm has adaptive step size control, and will backtrack and attempt a smaller parameter step if the nonlinear solve at some step fails to converge (Allgower and Georg, 2003). The step size increase is in part based on the number of Newton iterations that were required to converge the previous step, so a relatively easy nonlinear solve requiring just a handful of Newton iterations will lead to a more aggressive parameter step (see Salinger et al., 2002, for the detailed algorithm). We have found that starting with $\alpha_{0}=0$ leads to a system that will reliably converge from a trivial initial guess, that an initial step size of 0.1 is a good initial step, and that $\alpha_{\infty}=1$ provides an adequate stopping value.

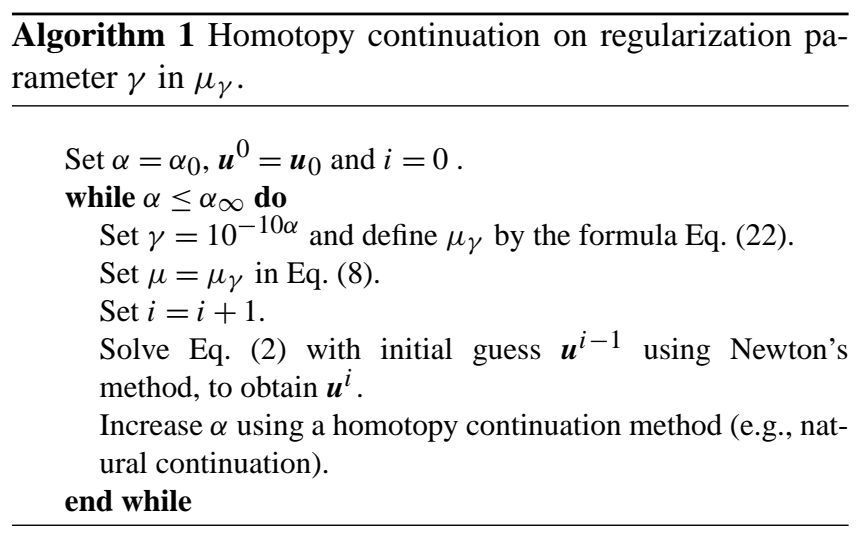

In general, the homotopy continuation approach leads to many fewer nonlinear solves than when the regularization parameter $\gamma$ in Eq. (22) is fixed to some small value, e.g., $\gamma=10^{-10}$, especially for problems where a "good" initial guess for Newton's method is unavailable. Moreover, with the homotopy continuation approach, it is found that a full step can often be employed in Newton's method line search algorithm, without the need for backtracking (i.e., iteratively reducing the step size in the line search algorithm).

We note that the homotopy continuation approach is in general effective when the initial guess is not close to the solution (in which case $\mu_{\gamma}$ is very small). Similarly, a good 
initial guess for $\boldsymbol{u}$ may not be a good initial guess when using continuation because the initial viscosity $\mu_{\gamma_{0}}$ for the continuation algorithm is generally far from the real viscosity $\mu$. When solving transient problems, it may be better to simply use a standard Newton method (without homotopy continuation), taking the solution at the previous time step as the initial guess, and using homotopy continuation only if the Newton solver has difficulties converging. A different approach, which may be used as an alternative to homotopy continuation, is to perform a few iterations using the Picard method and then to switch to the Newton method once the nonlinear iterations start to converge (e.g., Leng et al., 2015). The robustness and efficiency of the Newton solver with the homotopy continuation approach summarized in Algorithm 1 is studied numerically in Sect. 6.1.1.

\subsubsection{Multilevel preconditioning}

Multigrid preconditioners are among the most efficient and scalable linear solution techniques for resolving matrix equations associated with elliptic operators. The basic idea is to utilize multiple-resolution versions of the original problem to accelerate the iterative solution procedure. Toward this effect, smooth error components (in the current solution approximation) can be efficiently damped by applying a simple iterative process to a coarse-resolution version of the problem. This coarse version essentially facilitates the propagation of long-range information across the domain. Oscillatory components are effectively reduced through a simple iterative procedure, while smooth components are tackled using auxiliary lower-resolution versions of the problem. Different geometric multigrid methods have been successfully applied to the linear systems arising from ice sheet modeling simulations, e.g., Brown et al. (2013); Cornford et al. (2013); Isaac et al. (2014).

For our capability, we prefer algebraic multigrid (AMG) methods due to the potentially unstructured nature of the mesh in the horizontal plane. AMG methods have the advantage that the lower-resolution versions of the multigrid hierarchy are constructed automatically using only the matrix coefficient entries. Unfortunately, solution of the underlying linear systems is problematic due to the strong anisotropic nature of the discrete equations. This is essentially a consequence of the disparate scales in the horizontal and vertical directions and the associated large mesh aspect ratios. At the discrete level, these aspect ratios give rise to matrices where entries representing vertical coupling are generally much larger than entries representing horizontal coupling. Anisotropic phenomena within ice sheets and fairly different types of multigrid methods have been considered in recent prior works (Brown et al., 2013; Isaac et al., 2014; Jouvet and Graser, 2013).

From a multigrid perspective, reducing oscillatory errors in the horizontal direction is much more difficult than in the vertical direction. Furthermore, accurately capturing hor- izontal coupling on coarse levels can be challenging due to the relatively small size of the corresponding matrix entries (which are effectively averaged to generate the lowresolution versions). To avoid these difficulties, we have developed a hybrid structure/unstructured AMG multigrid capability that leverages the fact that our meshes, though unstructured in the horizontal plane, are structured in the vertical direction. That is, our 3-D meshes can be viewed as extrusions of unstructured 2-D meshes, allowing for varying vertical mesh spacing. A paper is in preparation to further describe the details of this hybrid algorithm. Here, we briefly describe its essence.

The basic concept behind the hybrid structured/unstructured AMG method is to first apply operatordependent multigrid semi-coarsening to initially coarsen the mesh and construct the first few levels of the multigrid hierarchy. Semi-coarsening and an operator-dependent multigrid both have a long history on structured grid problems (Dendy and Moulton, 2010; Schaffer, 1998; Brown et al., 2000). Semi-coarsening refers only to coarsening in some subset of coordinate directions and is often advocated to address anisotropic problems. Essentially, one only coarsens in directions where oscillatory errors are easily reduced. An operator-dependent multigrid refers to a family of algorithms that intimately takes advantage of the structure. They can be viewed as idealized or "perfect" grid transfers for one-dimensional (1-D) simplifications of the higher-dimensional problem. In this way, several coarselevel meshes are effectively constructed, each containing the same number of points within all horizontal planes. When it is no longer possible to further coarsen vertically (as there is just a single horizontal layer), a standard smoothed aggregation AMG method is applied to this horizontal problem, creating additional levels in the hierarchy. Thus, finer levels of the hierarchy are created via semi-coarsening and the operator-dependent multigrid (leveraging grid structure). Coarser levels are constructed via AMG, which is applied after the anisotropic behavior is no longer present (as there is just a single horizontal layer). To complete this brief description, we note that a line Jacobi method is used as the simple iterative scheme to damp oscillatory errors on the finer levels. It allows for aggressive semi-coarsening (i.e., reduction factors greater than 3 in the linear system dimension as one proceeds to progressively coarser levels). Polynomial smoothing is used on the levels associated with a standard AMG.

The algebraic multilevel preconditioner described above has been implemented in and is available through the (opensource) ML package of Trilinos (Heroux et al., 2005), in Trilinos 11.12 or later (see the Code Availability section at the end of this paper). The linear solver can be employed with or without the Albany and Albany/FELIX codes used to perform the ice sheet simulations described herein. The general $M L$ 
user's guide ${ }^{2}$ Gee et al. (2007) contains a detailed description of how to exercise the multigrid solver. Numerous example applications are included in the Trilinos release demonstrating how the multigrid solver can be used in different situations. An addendum to Gee et al. (2007) explaining how to invoke the particular software feature used in this paper (Tuminaro, 2014) describes how the multigrid semi-coarsening algorithm is specified from a user perspective. A paper is underway describing more algorithm details (Tuminaro et al., 2015).

\subsection{Software implementation}

The numerical methods described above are implemented in the Albany code base, an open-source ${ }^{3}$, multi-physics code/analysis package developed at Sandia National Laboratories. A full description of Albany can be found in a separate publication (Salinger et al., 2013). Briefly, Albany is a finite element code base for the solution and analysis of models of coupled PDEs using parallel, unstructured-grid, implicit algorithms. It makes use of numerous computational mathematics libraries from the Trilinos suite (Heroux et al., 2005 ), and has been previously used in other application domains such as quantum device modeling (Gao et al., 2013) and computational mechanics (Sun et al., 2013).

The software stack in Albany involves dozens of libraries that are delivered through Trilinos as independent software packages developed by small teams of domain experts. The Sierra ToolKit (STK) package is used for mesh database structures and mesh I/O. The Epetra package is used for distributed memory, parallel data structures for vectors and sparse matrices, which greatly simplify parallel operations such as halo exchanges for synchronizing data between processors. The Intrepid (Bochev et al., 2012) package provides flexible finite element discretization algorithms and general integration kernels. The PDE equations are described by a set of evaluation kernels whose evaluation is managed by the Phalanx package.

One of the main distinguishing characteristics of the $\mathrm{Al}$ bany code base is the use of the template-based generic programming (TBGP) approach (Pawlowski et al., 2012a, b). With this methodology, all that is required to implement a new set of physics in Albany is to code the residual of the PDE equations. Given this residual, Albany automatically computes and assembles the sparse Jacobian matrix and sensitivity vectors without any additional code development. TBGP makes extensive use of the Sacado package (Phipps et al., 2012) for automatic differentiation, which employs $\mathrm{C}++$ expression templates with operator overloading, and

\footnotetext{
${ }^{2}$ Available online at http://www.trilinos.org/oldsite/packages/ $\mathrm{ml} / \mathrm{mlguide} 5 . \mathrm{pdf}$.

${ }^{3}$ The Albany framework can be obtained from its public github repository by the interested reader: https:/github.com/ gahansen/Albany.
}

has been closely integrated with the Phalanx and Intrepid packages.

The Newton-based nonlinear system solver and homotopy continuation algorithm are implemented in the NOX (Pawlowski et al., 2006) and LOCA (Salinger et al., 2005) packages, respectively. These solvers can additionally perform sensitivity analysis using the analytic sensitivity vectors computed with automatic differentiation with respect to model parameters. Within the solvers, we have full runtime access to all the Trilinos preconditioners (ILU and algebraic multilevel preconditioners, from the Ifpack and $M L$ software packages, respectively) and linear solvers by specification in an input file. For the bulk of the computations in this paper, the $M L$ package was employed for algebraic multilevel preconditioners (Tuminaro, 2014), and the Belos package was employed for iterative solvers (CG or GMRES) (Bavier et al., 2012).

Albany is also coupled to the Dakota framework (Adams et al., 2009, updated 2013) of sampling-based optimization and UQ algorithms, which will play a significant role in model initialization, calibration, and projections. Although the application of optimization and UQ algorithms goes beyond the scope of this paper, we emphasize that the component-based approach for building this application code leads to the rapid incorporation of many sophisticated capabilities.

To give the reader an idea of how much time can be saved in writing a solver using modular packages or libraries, it is noted that it took one staff member working half-time for approximately six months to write the Albany/FELIX solver and to verify the code on the test cases presented in Sects. 45. It is estimated that all the work presented in the paper (including development of the AMG preconditioner based on semi-coarsening, described in Sect. 3.1.2) took approximately 1.5 FTEs (full-time equivalent units) worth of work.

\section{Verification using the method of manufactured solutions (MMS)}

We first conduct formal verification of the new $A l$ bany/FELIX code described in Sect. 3 through the method of manufactured solutions (MMS), using test cases derived here explicitly for this purpose. A survey of the literature reveals that past work has focused on deriving MMS benchmarks for the "shallow ice" and nonlinear Stokes models (e.g., Bueler et al., 2007; Leng et al., 2013, respectively) rather than the FO approximation Eq. (2). The lack of MMS solutions for the FO Stokes equations in the literature is likely due to the complexity of these equations, which makes deriving source terms for a given manufactured solution difficult, if not intractable. Here, we derive some new MMS benchmarks for simplified versions of the FO Stokes Eq. (2) in 2-D. These equations are obtained by neglecting gradients in one of the coordinate directions, first $z$ (Sect. 4.1), then $y$ (Sect. 4.2), and allow us to look at the convergence of our computed so- 
lution to an exact solution for parts of the governing PDEs. The terms appearing in our test cases are simple enough to be implemented by anyone simply by referring to the expressions in this paper. It is emphasized that the test cases are intended to be used as part of a multi-stage code verification that also includes verification of the 3-D FO Stokes equations using code-to-code comparisons and mesh convergence studies on realistic geometries (Sects. 5 and 6, respectively).

Here, we use the Albany/FELIX code and these new MMS benchmarks to verify (i) that the dynamics have been implemented correctly, and (ii) that the type of finite elements employed show convergence at their expected theoretical rates.

We consider four different finite element types in our numerical convergence study: three node triangles (denoted by "Tri 3"), four node quadrilaterals (denoted by "Quad 4"), six node triangles (denoted by "Tri 6"), and nine node quadrilaterals (denoted by "Quad 9") (Fig. 1). Convergence is evaluated in the discrete $l^{2}$ norm. In particular, the relative error in a computed solution, denoted by $\mathcal{E}_{\text {rel }}^{\text {disc }}$, is calculated from

$\mathcal{E}_{\text {rel }}^{\text {disc }}=\frac{\left\|\boldsymbol{u}_{n}-\boldsymbol{u}\right\|_{2}}{\|\boldsymbol{u}\|_{2}}$,

where $\|\cdot\|_{2}$ denotes the discrete $l^{2}$ norm, $\boldsymbol{u}^{T} \equiv(u, v)$ is the exact solution to Eq. (24), and $\boldsymbol{u}_{n}$ is the numerically computed solution to Eq. (24). It is well known from classical finite element theory (Hughes, 2000) that the theoretical convergence rate in the norm considered is 2 for the Tri 3 and Quad 4 elements, and 3 for the Quad 6 and Quad 9 elements. Hence, the first two elements are referred to as first-order finite elements and the second two elements are referred to as second-order finite elements. Note that the quadrilateral elements are expected to deliver a more accurate solution than their triangular counterparts of the same order.

\section{1 $x-y$ MMS test case}

Consider the FO Stokes Eq. (2) in 2-D, that is, Eq. (2) with all the $\frac{\partial}{\partial z}$ terms neglected. Assume these equations are posed on a domain whose sides are aligned with the $x$ and $y$ axes in a Cartesian reference frame, so that $\frac{\partial s}{\partial x}=\frac{\partial s}{\partial y}=0$. Let $\mathbf{f}^{T} \equiv$ $\left(f_{1}, f_{2}\right)$ be a source term for Eq. (2), to be determined such that a given manufactured solution satisfies these equations. Under these assumptions, the FO Stokes system Eq. (2) has the following form:

$$
\left\{\begin{array}{c}
-\frac{\partial}{\partial x}\left(4 \mu_{2-\mathrm{D}, x y} \frac{\partial u}{\partial x}+2 \mu_{2-\mathrm{D}, x y} \frac{\partial v}{\partial y}\right) \\
-\frac{\partial}{\partial y}\left(\mu_{2-\mathrm{D}, x y} \frac{\partial u}{\partial y}+\mu_{2-\mathrm{D}, x y} \frac{\partial v}{\partial x}\right)+f_{1}=0, \\
-\frac{\partial}{\partial x}\left(\mu_{2-\mathrm{D}, x y} \frac{\partial u}{\partial x}+\mu_{2-\mathrm{D}, x y} \frac{\partial v}{\partial y}\right) \\
-\frac{\partial}{\partial y}\left(2 \mu_{2-\mathrm{D}, x y} \frac{\partial u}{\partial x}+4 \mu_{2-\mathrm{D}, x y} \frac{\partial v}{\partial y}\right)+f_{2}=0,
\end{array}\right.
$$

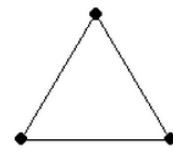

(a)

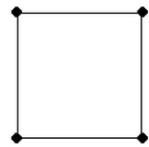

(b)

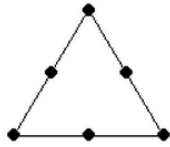

(c)

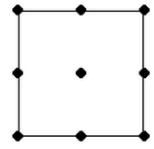

(d)
Figure 1. 2-D finite elements evaluated in the manufactured solution test cases. (a) Tri 3, (b) Quad 4, (c) Tri 6, (d) Quad 9.

where the viscosity $\mu_{2-\mathrm{D}, x y}$ is given by the 2-D version of Eq. (8):

$$
\begin{aligned}
\mu_{2-\mathrm{D}, x y} & =\frac{1}{2} A^{-\frac{1}{n}}\left\{\left(\frac{\partial u}{\partial x}\right)^{2}+\left(\frac{\partial v}{\partial y}\right)^{2}+\frac{\partial u}{\partial x} \frac{\partial v}{\partial y}\right. \\
& \left.+\frac{1}{4}\left(\frac{\partial u}{\partial y}+\frac{\partial v}{\partial x}\right)^{2}\right\}^{\left(\frac{1}{2 n}-\frac{1}{2}\right)} \cdot
\end{aligned}
$$

We note that the $x-y$ FO Stokes Eq. (24) can be viewed as a test for ice shelves; stress gradients in the $x-z$ plane are negligible compared to those in the $x-y$ plane.

The $x-y$ MMS first test case is posed on a box domain, namely $\Omega=(0,1) \times(0,1)$ with Robin boundary conditions on $\partial \Omega$. The source term in Eq. (24) is derived such that the exact solution to this system is given by the following expression:

$u=e^{x} \sin (2 \pi y)$,

$v=e^{x} \cos (2 \pi y)$,

(Fig. 2). Substituting Eq. (26) into Eq. (24), the source terms $f_{1}$ and $f_{2}$ are obtained:

$$
\begin{aligned}
f_{1} & =2 \mu_{2-\mathrm{D}, x y} e^{x} \sin (2 \pi y)\left[2-3 \pi-2 \pi^{2}\right] \\
& +A^{-\frac{1}{n}}\left(\frac{1}{n}-1\right) \dot{\epsilon}_{\mathrm{e}, 2-\mathrm{D}}^{\frac{1}{n}-2}\left(\frac{\partial \dot{\epsilon}_{\mathrm{e}, 2-\mathrm{D}}}{\partial x}\left(2 \dot{\epsilon}_{x x}+\dot{\epsilon}_{y y}\right)\right. \\
& \left.+\frac{\partial \dot{\epsilon}_{\mathrm{e}, 2-\mathrm{D}}}{\partial y} \dot{\epsilon}_{x y}\right) \\
f_{2} & =2 \mu_{2-\mathrm{D}, x y} e^{x} \cos (2 \pi y)\left[3 \pi+\frac{1}{2}-8 \pi^{2}\right] \\
& +A^{-\frac{1}{n}}\left(\frac{1}{n}-1\right) \dot{\epsilon}_{\mathrm{e}, 2-\mathrm{D}}^{\frac{1}{n}-2}\left(\frac{\partial \dot{\epsilon}_{\mathrm{e}, 2-\mathrm{D}}}{\partial x} \dot{\epsilon}_{x y}\right. \\
& \left.+\frac{\partial \dot{\epsilon}_{\mathrm{e}, 2-\mathrm{D}}}{\partial y}\left(\dot{\epsilon}_{x x}+2 \dot{\epsilon}_{y y}\right)\right)
\end{aligned}
$$

where

$$
\begin{array}{r}
\dot{\epsilon}_{e, 2-D} \equiv \sqrt{\left(\frac{\partial u}{\partial x}\right)^{2}+\left(\frac{\partial v}{\partial y}\right)^{2}+\frac{\partial u}{\partial x} \frac{\partial v}{\partial y}+\frac{1}{4}\left(\frac{\partial u}{\partial y}+\frac{\partial v}{\partial x}\right)^{2}} \\
=e^{x} \sqrt{\left(1+4 \pi^{2}-2 \pi\right) \sin ^{2}(2 \pi y)+\frac{1}{4}(2 \pi+1)^{2} \cos ^{2}(2 \pi y)},
\end{array}
$$




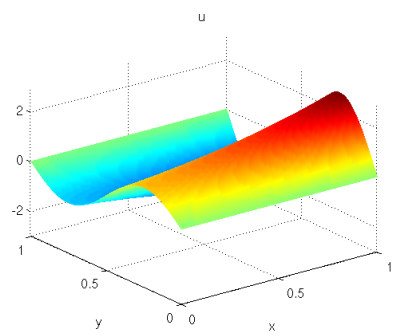

(a)

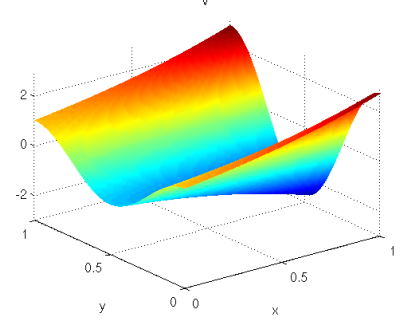

(b)
Figure 2. Plots of exact solutions to the $x-y$ MMS test case: (a) $u$, (b) $v$.

and $\mu_{2-\mathrm{D}, x y}$ is given by Eq. (25). The solution (26) implies the following boundary conditions on the boundary of $\Omega$ :

$$
\begin{aligned}
& \dot{\boldsymbol{\epsilon}}_{1} \cdot \boldsymbol{n}=2(\pi-1) u, \dot{\boldsymbol{\epsilon}}_{2} \cdot \boldsymbol{n}=-\left(\pi+\frac{1}{2}\right) v, \text { at } x=0, \\
& \dot{\boldsymbol{\epsilon}}_{1} \cdot \boldsymbol{n}=-2(\pi-1) u, \dot{\boldsymbol{\epsilon}}_{2} \cdot \boldsymbol{n}=\left(\pi+\frac{1}{2}\right) v, \text { at } x=1, \\
& u=0, \dot{\boldsymbol{\epsilon}}_{2} \cdot \boldsymbol{n}=0, \text { at } y=0 \text { and } y=1, \\
& v=0, \text { at }(x, y)=(0,0)
\end{aligned}
$$

where $\boldsymbol{n}$ denotes the outward unit normal vector to a given boundary and where

$\dot{\boldsymbol{\epsilon}}_{1}^{T}=\left(2 \dot{\epsilon}_{x x}+\dot{\epsilon}_{y y}, \dot{\epsilon}_{x y}\right) \in \mathbb{R}^{2}$

and

$\dot{\boldsymbol{\epsilon}}_{2}^{T}=\left(\dot{\epsilon}_{x y}, \dot{\epsilon}_{x x}+2 \dot{\epsilon}_{y y}\right) \in \mathbb{R}^{2}$

The last condition on Eq. (30) is imposed to guarantee uniqueness of the $v$ component of the velocity vector.

For the $x-y$ MMS test case considered here, the values of the flow rate factor and Glen's flow law exponent were taken to be $A=1$ and $n=3$, respectively. The relative errors Eq. (23) as a function of the mesh size $h$ for the $x-y$ MMS test case are plotted on a log-log plot in Fig. 3. The two lowest-order finite elements (Tri 3 and Quad 4) converge at their theoretical rates of 2, whereas the higher-order finite elements (Tri 6 and Quad 9) exhibit a slight superconvergence over their theoretical convergence rate of 3 . As expected, the quadrilateral elements deliver a more accurate solution than their triangular counterparts.

\section{2 $x-z$ MMS test case}

The 2-D FO Stokes equations in the $x-z$ variables are obtained from Eq. (2) by neglecting the $y$-component of the velocity $(v)$ and all the $\frac{\partial}{\partial y}$ terms. The vector $\dot{\boldsymbol{\epsilon}}_{1}^{T}$ reads

$\dot{\boldsymbol{\epsilon}}_{1}^{T}=\left(2 \dot{\epsilon}_{x x}, \dot{\epsilon}_{x z}\right)$,

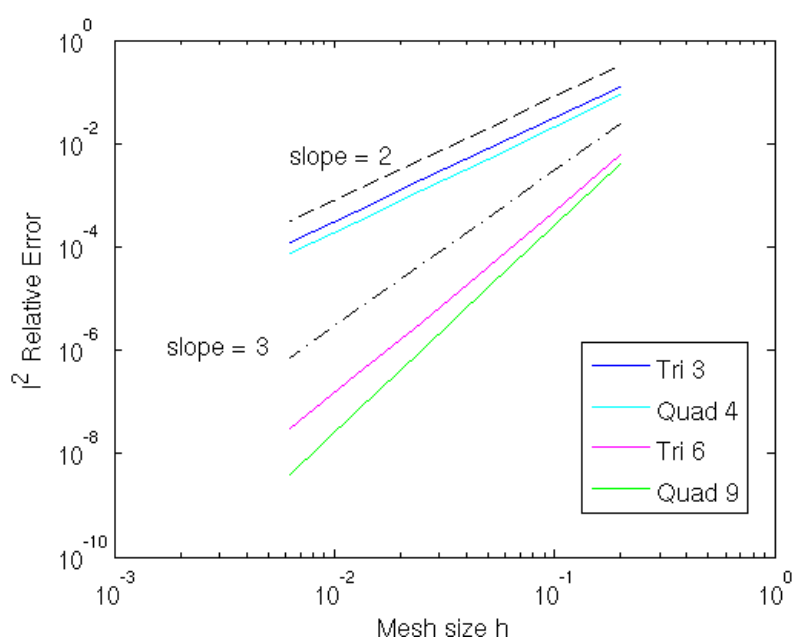

Figure 3. Convergence rates for the $x-y$ MMS test case in the discrete $l^{2}$ norm Eq. (23).

and the FO Stokes equations reduce to the following 2-D equation in the $x-z$ plane:

$-\frac{\partial}{\partial x}\left(4 \mu_{2-\mathrm{D}, x z} \frac{\partial u}{\partial x}\right)-\frac{\partial}{\partial z}\left(\mu_{2-\mathrm{D}, x z} \frac{\partial u}{\partial z}\right)+f_{1}=0$,

where

$\mu_{2-\mathrm{D}, x z}=\frac{1}{2} A^{-1 / n}\left(\dot{\epsilon}_{x x}^{2}+\dot{\epsilon}_{x z}^{2}\right)^{\frac{1}{2 n}-\frac{1}{2}}, \quad f_{1}=\rho g \frac{\partial s}{\partial x}$.

We consider the following approximate solution of Eq. (34):

$$
\begin{aligned}
u & =\frac{2 A \rho^{n} g^{n}}{n+1}\left((s-z)^{n+1}-H^{n+1}\right)\left|\frac{\partial s}{\partial x}\right|^{n-1} \frac{\partial s}{\partial x} \\
& -\frac{\rho g}{\beta} H \frac{\partial s}{\partial x} .
\end{aligned}
$$

The first term of $u$ is the solution of the SIA with no-slip at the bedrock interface, whereas the second term is the solution of the SSA equation when $H$ and $\beta$ are constant and $s$ is quadratic in $x$. We now use the solution $u$ as our manufactured solution, and we modify the forcing term $f_{1}$ of Eq. (34) so that the FO equation is exactly satisfied by $u$. In particular, we set $n=3$, and consider the geometry defined by $s=s_{0}-\alpha x^{2}, b=s-H, x \in(-L, L)$, with constants $s_{0}, \alpha, H$, and $\beta$. Then,

$$
\begin{gathered}
f_{1}=\frac{16}{3} A \mu_{2-\mathrm{D}, x z}^{4}\left(-2 \phi_{4}^{2} \phi_{5}+24 \phi_{3} \phi_{4}\left(\phi_{1}+2 \alpha x^{2}\right)\right. \\
\left.-6 x^{3} \phi_{1}^{3} \phi_{2} \phi_{3}-18 x^{2} \phi_{1}^{2} \phi_{2} \phi_{4}^{2}-6 x \phi_{1} \phi_{3} \phi_{5}\right),
\end{gathered}
$$


where

$$
\begin{aligned}
& \phi_{1}=z-s, \quad \phi_{2}=4 A \alpha^{3} \rho^{3} g^{3} x, \quad \phi_{3}=4 x^{3} \phi_{1}^{5} \phi_{2}^{2}, \\
& \phi_{4}=8 \alpha x^{3} \phi_{1}^{3} \phi_{2}-\frac{2 H \alpha \rho g}{\beta}+3 x \phi_{2}\left(\phi_{1}^{4}-H^{4}\right), \\
& \phi_{5}=56 \alpha x^{2} \phi_{1}^{3} \phi_{2}+48 \alpha^{2} x^{4} \phi_{1}^{2} \phi_{2}+6 \phi_{2}\left(\phi_{1}^{4}-H^{4}\right), \\
& \mu_{2-\mathrm{D}, x z}=\frac{1}{2}\left(A \phi_{4}^{2}+A x \phi_{1} \phi_{3}\right)^{-\frac{1}{3}} .
\end{aligned}
$$

Boundary conditions are of the Robin and Neumann type, and are given by

$\dot{\boldsymbol{\epsilon}}_{1} \cdot \boldsymbol{n}=f_{\text {top }}$, at $z=s(x)$,

$\dot{\boldsymbol{\epsilon}}_{1} \cdot \boldsymbol{n}+\beta u=f_{\text {bed }}$, at $z=b(x)$,

$\dot{\boldsymbol{\epsilon}}_{1} \cdot \boldsymbol{n}=f_{\text {lat }}$, at $x=L$,

$\dot{\boldsymbol{\epsilon}}_{1} \cdot \boldsymbol{n}=-f_{\text {lat }}$, at $x=-L$,

where

$$
\begin{aligned}
f_{\text {lat }} & =-4 \phi_{4} \mu_{2-\mathrm{D}, x z}, \\
f_{\text {top }} & =-4 \phi_{4} \mu_{2-\mathrm{D}, x z} n_{x}^{\text {top }}-4 \phi_{2} x^{2} \phi_{1}^{3} \mu_{2-\mathrm{D}, x z} n_{y}^{\text {top }}, \\
f_{\text {bed }} & =-4 \phi_{4} \mu_{2-\mathrm{D}, x z} n_{x}^{\text {bed }}-4 \phi_{2} x^{2} \phi_{1}^{3} \mu_{2-\mathrm{D}, x z} n_{y}^{\text {bed }} \\
& +2 H \alpha \rho g x-\beta x^{2} \phi_{2}\left(\phi_{1}^{4}-H^{4}\right) .
\end{aligned}
$$

Here, the components of the normal to the top and bedrock surfaces read $n_{x}^{\text {top }}=\frac{2 \alpha x}{\sqrt{4 \alpha^{2} x^{2}+1}}, n_{z}^{\text {top }}=\frac{1}{\sqrt{4 \alpha^{2} x^{2}+1}}$ and $n_{x}^{\text {top }}=$ $-n_{x}^{\text {bed }}, n_{z}^{\text {top }}=-n_{z}^{\text {bed }}$

Figure 4 shows a contour plot of the exact solution to the $x-z$ MMS problem Eq. (36) and the domain $\Omega$ on which this problem is posed.

Reasonable values for the constants defining the $x-z$ MMS test case, and the ones used here, are $L=50 \mathrm{~km}$, $s_{0}=2 \mathrm{~km}, H=1 \mathrm{~km}, \alpha=4 e-5 \mathrm{~km}^{-1}, \beta=1 \mathrm{kPa} \mathrm{yr} \mathrm{m}^{-1}$ and $A=10^{-4} \mathrm{k}^{-4} \mathrm{~Pa}^{-3} \mathrm{a}^{-1}$.

Figure 5 plots the relative errors in Eq. (23) on a log-log scale as a function of the horizontal mesh resolution $h_{x}$ for the $x-z$ MMS test case. The $x$ and $z$ resolutions considered are such that $n=5,10,20$, and 40, where $n$ denotes the number of elements in each spatial direction. The two first-order elements, Tri 3 and Quad 4, converge at a rate of 2, their theoretical convergence rate. The convergence rate of the Tri 6 element is close to its theoretical convergence rate of 3 . The Quad 9 element exhibits a slight superconvergence over its theoretical convergence rate of 3 . As for the $x-y$ MMS test case considered in Sect. 4.1, and as expected, the quadrilateral elements deliver a more accurate solution than their triangular analogs.

\section{Intercomparison with other codes and benchmarks}

In this section, we discuss further (informal) verification of results for Albany/FELIX using some canonical ice sheet benchmarks, namely the ISMIP-HOM tests A and C

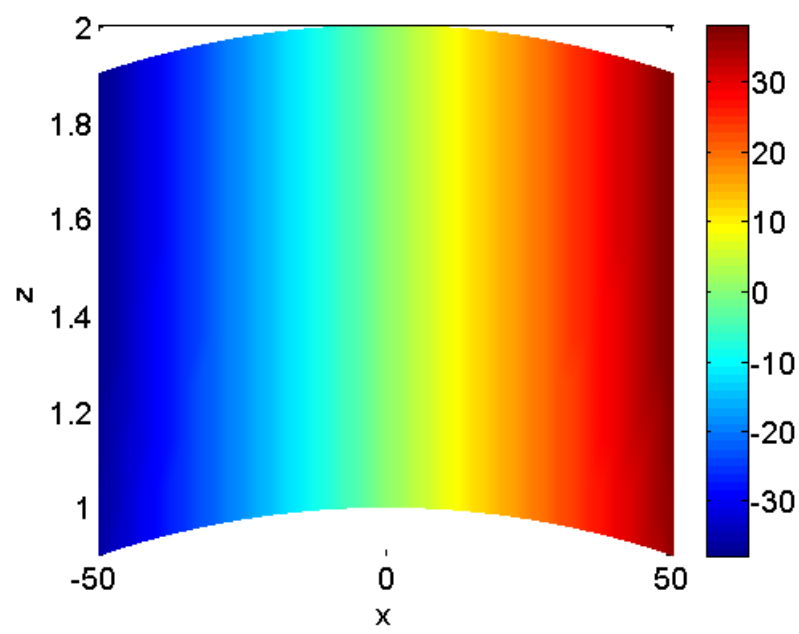

Figure 4. Contour plot of the exact solution to the $x-z$ MMS test case.

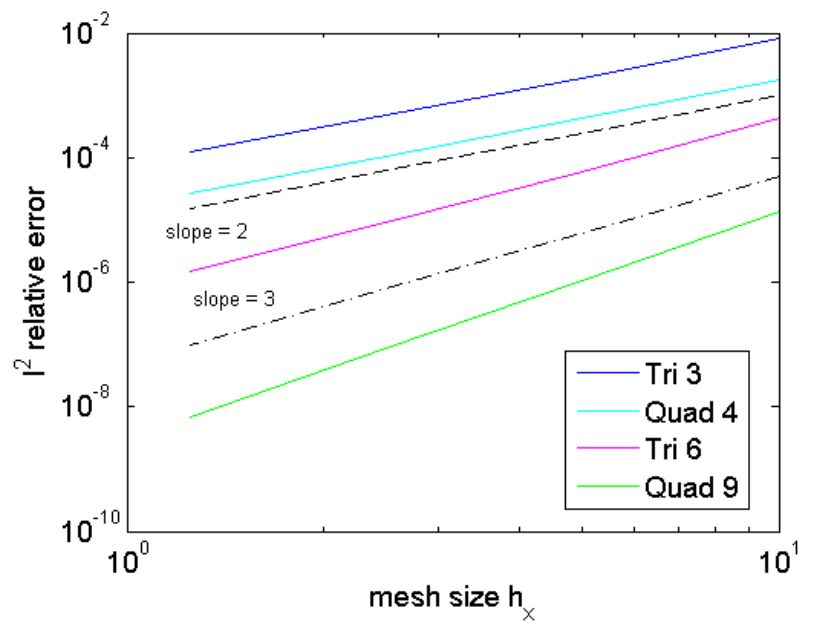

Figure 5. Convergence rates for the $x-z$ MMS test case in the discrete $l^{2}$ norm Eq. (23).

(Sect. 5.1), and the confined shelf test case (Sect. 5.2) (Rommelaere, 1996). For these problems, the exact solution is not known in closed analytic form and our quasi-verification consists of code-to-code comparisons between the solution computed in Albany/FELIX, the results from other models participating in the original benchmark experiments, and the FO approximation, the finite element code of Perego et al. (2012).

The values of the physical parameters used in the two test cases considered are summarized in Table 1. We note that the units employed in our implementation are $\mathrm{m} \mathrm{a}^{-1}$ for the ice velocities $u$ and $v$ (where "a" denotes years) and $\mathrm{km}$ for the length scale (e.g., the mesh dimensions). Our units are the same as in Perego et al. (2012), but differ from other implementations, which often use a length scale of meters (m). Our units give rise to matrices with smaller differences 
in scale (which may be better scaled), as there is in general a smaller difference in scale in the relevant parameter values (e.g., $A=10^{-4} \mathrm{k}^{-(n+1)} \mathrm{Pa}^{-n} \mathrm{a}^{-1}$ when the mesh is in km vs. $A=10^{-16} \mathrm{~Pa}^{-n} \mathrm{a}^{-1}$ when the mesh is in $\mathrm{m}$, where $\mathrm{k}=\mathrm{km} \mathrm{m}^{-1}=10^{3}$ ).

\subsection{ISMIP-HOM benchmarks}

The ISMIP-HOM test cases (Pattyn et al., 2008) are a canonical set of benchmark experiments for so-called "higherorder" ice sheet models. Here, we consider tests A and C, both of which are specified on a horizontal, periodic domain with a unit length of $L \mathrm{~km}$. The bedrock surface, $\Gamma_{b}$, is given by a continuous function $z=b(x, y) \in \mathbb{R}^{2}$ and the upper surface, $\Gamma_{s}$, is given by a continuous function $z=s(x, y) \in \mathbb{R}^{2}$. The geometries are generated from a uniform hexahedral mesh of the unit cube $(0,1)^{3} \in \mathbb{R}^{3}$ via the following transformation:

$x=L X, \quad y=L Y, \quad z=s(x, y) Z+b(x, y)(1-Z)$,

where $X, Y$, and $Z$ are the coordinates of the unit cube (in $\mathrm{km}$ ), and $L \in \mathbb{N}$ is given. That is, a uniform mesh of $n_{x} \times n_{y} \times n_{z}$ elements is first generated of $(0,1)^{3}$, to yield the nodal coordinates $X, Y$, and $Z$; then, the transformation Eq. (41) is applied. The following domain sizes are considered: $L=5,10,20,40,80$ and $160 \mathrm{~km}$. Each domain is discretized using an $80 \times 80 \times 20$ mesh of hexahedral elements. As a part of the quasi-verification, the Albany/FELIX solution is compared with the solution computed in the finite element code of Perego et al. (2012) at the upper surface along the line $y=L / 4$. Table 2 shows the relative difference between the Albany/FELIX and Perego et al. (2012) solutions in the $l^{2}$ norm along this line, calculated from the formula Eq. (23) with the Perego et al. (2012) solution taken as the reference solution. Differences in the solutions are likely due to the different finite elements used: trilinear finite elements on hexahedra are used in Albany/FELIX, whereas linear finite elements on tetrahedra are used in the code of Perego et al. (2012).

\subsubsection{ISMIP-HOM test A}

The first ISMIP-HOM benchmark considered is test A. For this problem, the upper ice surface boundary $\left(\Gamma_{S}\right)$ is given by the following linear function

$s(x, y)=-x \tan \alpha$,

and the bedrock boundary $\left(\Gamma_{b}\right)$ is given by the following trigonometric function

$b(x, y)=s(x, y)-1+\frac{1}{2} \sin \left(\frac{2 \pi}{L} x\right) \sin \left(\frac{2 \pi}{L} y\right)$,

with $\alpha=0.5^{\circ}$. The geometry is thus that of a uniformly sloping slab along the $x$ coordinate direction with a doubly periodic, "egg crate" shaped bed. A no-slip boundary condition
Table 2. Relative differences between Albany/FELIX and Perego et al. (2012) solutions for ISMIP-HOM tests A and C.

\begin{tabular}{lll}
\hline$L(\mathrm{~km})$ & Test A & Test C \\
\hline 5 & $0.00735 \%$ & $0.386 \%$ \\
10 & $0.00629 \%$ & $0.248 \%$ \\
20 & $0.00132 \%$ & $0.176 \%$ \\
40 & $0.00408 \%$ & $0.213 \%$ \\
80 & $0.0407 \%$ & $0.277 \%$ \\
160 & $0.127 \%$ & $0.320 \%$ \\
\hline
\end{tabular}

is prescribed on $\Gamma_{b}$ (with $\Gamma_{0} \equiv \Gamma_{b}$ and $\Gamma_{\beta}=\varnothing$ ), stress-free boundary conditions are prescribed on the upper surface $\Gamma_{s}$, and periodic boundary conditions are prescribed on the lateral boundaries $\Gamma_{1}$.

Figure 6 compares the solution computed within the $A l$ bany/FELIX code for ISMIP-HOM test A with the solution computed by the code of Perego et al. (2012) (denoted by MP12 in this figure). The agreement between the two is excellent. The second column of Table 2 reports the relative difference between these two solutions in the $l^{2}$ norm Eq. (23). The relative difference is at most $0.1 \%$ for $L=180$ and on the order of $0.001 \%$ for $L=5,10,20$, and 40 .

Figure 6 also includes the mean and standard deviation of solutions computed by other models participating in the original set of benchmark experiments. For a detailed description of these models, the reader is referred to Pattyn et al. (2008). For all values of $L$ considered, the Albany/FELIX solution is within 1 standard deviation of the mean of the other FO models considered in the original set of experiments. In Fig. 6, the solutions labeled "Full Stokes" were calculated using the (more expensive but more physically realistic) full Stokes model for ice sheet flow (detailed in Appendix A). Comparing a FO Stokes solution to the full Stokes solution reveals how well the FO Stokes physics approximate the full Stokes model. The reader can observe by examining Fig. 6 that agreement between the FO Stokes and the full Stokes solutions improves with increasing $L$.

\subsubsection{ISMIP-HOM test C}

For ISMIP-HOM test C, the upper and bedrock surfaces $\left(\Gamma_{S}\right.$ and $\Gamma_{b}$, respectively) are given by the following linear functions:

$s(x, y)=-x \tan \alpha, \quad b(x, y)=s(x, y)-1$,

with $\alpha=0.1^{\circ}$. In addition to having a different geometry than test $\mathrm{A}$, test $\mathrm{C}$ also differs in the boundary conditions. Unlike test $\mathrm{A}$, sliding boundary conditions are prescribed on the bedrock $\left(\Gamma_{\beta} \equiv \Gamma_{b}\right.$ and $\left.\Gamma_{0} \equiv \varnothing\right)$, with the basal sliding coefficient given by

$\beta(x, y)=1+\sin \left(\frac{2 \pi}{L} x\right) \sin \left(\frac{2 \pi}{L} y\right)$. 


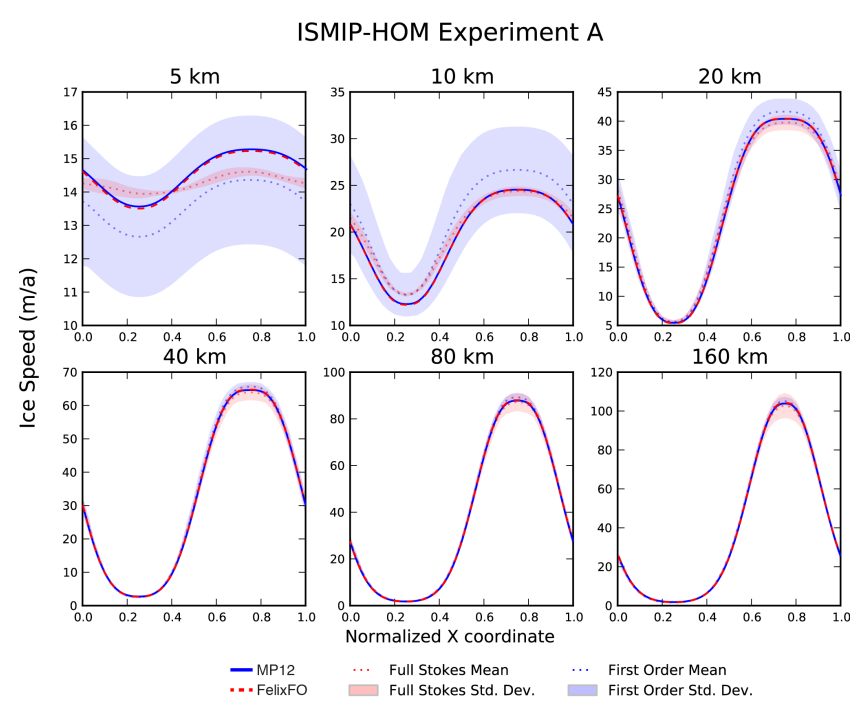

Figure 6. ISMIP-HOM test A: surface velocity component $u$ as a function of $x$ at $y=L / 4$ for each $L$ considered. The blue solid line (MP12) represents results from Perego et al. (2012) and the red dashed line (labeled FelixFO) represents results from the current solver.

The boundary conditions at the upper and lateral boundaries $\left(\Gamma_{s}\right.$ and $\Gamma_{1}$, respectively) are the same as for test A, namely stress-free and periodic, respectively. The geometry is thus that of a constant thickness, uniformly sloping slab along the $x$ coordinate direction with a doubly periodic, "egg crate" spatial pattern for the basal friction parameter $\beta$.

The test case solution computed in Albany/FELIX is shown in Fig. 7, along with the solution computed using the solver of Perego et al. (2012). For every $L$ considered, the relative difference between Albany/FELIX and the solver of Perego et al. (2012) (denoted, as before, by MP12 in Fig. 7) is less than $1 \%$ (Table 2). Moreover, as for ISMIP-HOM test A, the Albany/FELIX solution is within 1 standard deviation of the model means for each value of $L$. As for ISMIPHOM test A, Fig. 7 also illustrates how well the FO Stokes model compares to the (more expensive but more accurate) full Stokes model. As for test A, the two models agree better for larger $L$.

\subsection{Confined shelf benchmark}

We next consider an idealized ice shelf test case, referred to here as the "confined shelf" test case, which is a slightly modified version of test 3 from the Ice Shelf Model Intercomparison exercise (Rommelaere, 1996). The geometry is that of a $500 \mathrm{~m}$ thick slab of ice with equal extents of $200 \mathrm{~km}$ along the $x$ and $y$ dimensions, floating in hydrostatic equilibrium. A stress-free boundary condition is applied at the upper and basal boundaries ( $z=s$ and $z=b$, respectively) and homogeneous Dirichlet boundary conditions $(u=v=0)$ are applied on three of the four lateral boundaries (the eastern

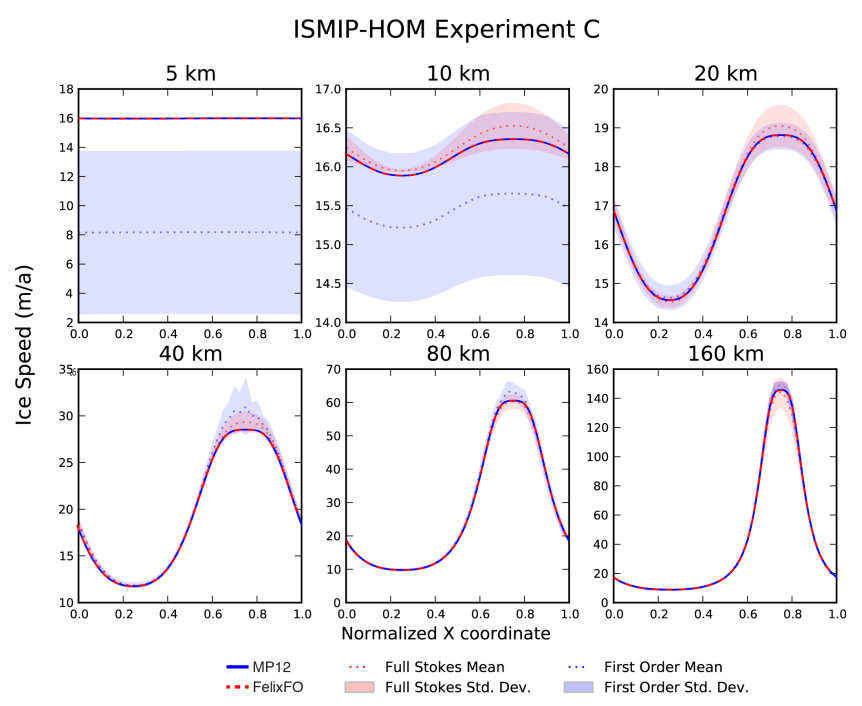

Figure 7. ISMIP-HOM test C: surface velocity component $u$ as a function of $x$ at $y=L / 4$ for each $L$ considered. The blue solid line (MP12) represents results from Perego et al. (2012) and the red dashed line (labeled FelixFO) represents results from the current solver. Note that, for the $5 \mathrm{~km}$ test, the MP12 and FelixFO results directly overly the results for the full Stokes models participating in the original intercomparison.

$(x=200)$, western $(x=0)$ and northern $(y=200)$ boundaries). The southern $(y=0)$ lateral boundary is open to the ocean and subject to the open-ocean Neumann boundary condition described in Sect. 2 (boundary condition c). The values of the parameters that appear in Eq. (18) can be found in Table 1.

The confined shelf geometry is discretized using a structured tetrahedral mesh of $41 \times 41$ nodes in the $x-y$ plane with 10 vertical levels. As with the ISMIP-HOM test cases, the solution for the confined shelf test case computed in our code, Albany/FELIX, is compared to the solution computed by the solver of Perego et al. (2012) on the same mesh. Figure 8 shows the solution calculated in Albany/FELIX, which is visually identical to the solution computed by the solver of Perego et al. (2012). The difference between the Albany/FELIX and Perego et al. (2012) solutions was found to be on the order of $\mathcal{O}\left(10^{-10}\right)$ at all grid points.

\section{Convergence study using realistic geometry}

The final results presented herein are the results of a numerical convergence and performance study using a realistic, $1 \mathrm{~km}$ spatial resolution Greenland Ice Sheet (GIS) geometry (i.e., surface and bed topography from Bamber et al., 2013).

First, we present results from a 3-D mesh convergence study in which a set of uniform quadrilateral meshes of different horizontal and vertical resolutions were considered. We began by generating a quadrilateral mesh with an $8 \mathrm{~km}$ 


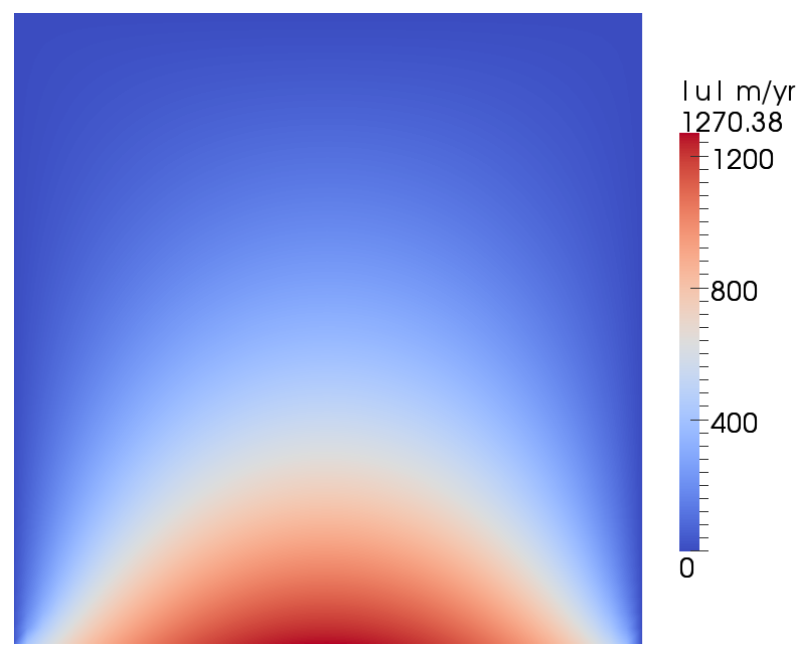

Figure 8. Albany/FELIX solution to the confined-shelf test case (indistinguishable from the solution obtained by the solver of Perego et al. (2012)).

horizontal resolution. We then refined this coarse mesh uniformly in the horizontal direction (by splitting each quadrilateral finite element into four smaller quadrilaterals; see Fig. 9) four times to yield meshes with resolutions of 4,2 , and $1 \mathrm{~km}$, and $500 \mathrm{~m}$. The horizontal meshes were then extruded into 3-D hexahedral meshes with uniform or graded spacing between the vertical layers. In the graded vertical spacing case, a transformation is performed such that a mesh with $n_{z}$ vertical layers is finer near the bedrock boundary $\Gamma_{b}$ and becomes progressively coarser moving up, towards the surface boundary $\Gamma_{s}$. The formulas ${ }^{4}$ for the coordinate of the $i$ th vertical layer, $z_{i}$ (for $i=0, \ldots, n_{z}$, where $n_{z}$ is the number of vertical layers), for each of these two spacings, is given in Table 3.

The number of layers considered in our study ranges from 5 to 80 . Realistic basal friction coefficient $(\beta)$ fields were calculated by solving a deterministic inversion problem that minimizes simultaneously the discrepancy between modeled and observed surface velocities, modeled and observed bed topography, and between a specified surface mass balance field and the modeled flux divergence (see Perego et al., 2014, for more details). A realistic, 3-D temperature field, originally calculated using CISM for the study in Shannon et al. (2013), was included as an initial condition in order to provide realistic values for the flow-law rate factor Eq. (10). Prior to being interpolated onto the meshes at hand, the original topography, surface height, basal friction and temperature data were smoothed by convolution with a 2-D Gaussian filter (with a standard deviation of $5 \mathrm{~km}$ ). This smoothing filter reduces the small-scale variations of the original fields, so that it is reasonable to consider meshes from $8 \mathrm{~km}$ to $500 \mathrm{~m}$

\footnotetext{
${ }^{4}$ The formula for the graded $z$ spacing can be found in the CISM documentation (Price et al., 2015), available at http://oceans11.lanl. gov/cism.
}

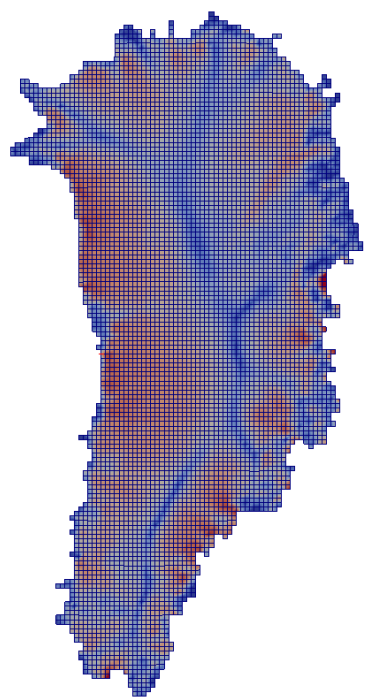

(a)

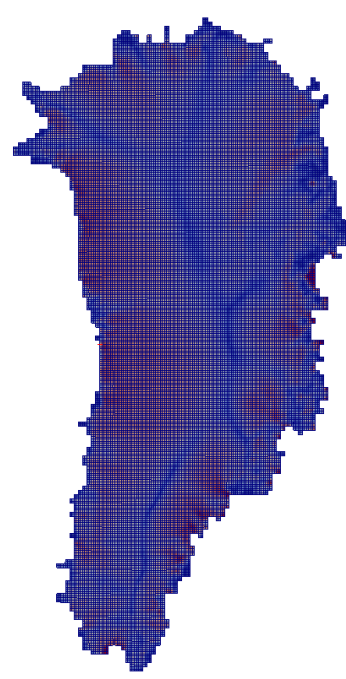

(b)
Figure 9. Examples of uniform mesh refinement: (a) no refinement (8 km GIS), and (b) one level of refinement (4 km GIS).

Table 3. Formulas for different vertical mesh-spacing strategies (uniform vs. graded), for $i=0, \ldots, n_{z}$.

\begin{tabular}{ll}
\hline$z$ spacing & $z_{i}$ \\
\hline Uniform & $\frac{i}{n_{z}}$ \\
Graded & $1-\frac{4}{3}\left[1-\left(\frac{n_{z}}{2 n_{z}-i}\right)^{2}\right]$ \\
\hline
\end{tabular}

for our convergence study. Using directly the non-smoothed data, we would have needed to consider much finer meshes in order to obtain asymptotic convergence.

The purpose of our GIS mesh convergence study is threefold:

(i) to show a theoretical convergence rate for the finite elements evaluated with respect to refinement in all three coordinate directions,

(ii) to determine in a rigorous fashion for a GIS problem with a fixed horizontal mesh resolution how many vertical layers are required to achieve a solution with a desired accuracy, and

(iii) to investigate whether the performance of our linear and nonlinear solvers changes with the number of vertical layers.

From finite element theory, theoretical convergence rates are expected for a problem in which the data are fixed on all meshes considered, so better-resolved data are intentionally not introduced on the coarser meshes that were part of our convergence study in this section. A high-resolution 
GIS problem, with real, high-resolution data is considered in Sect. 6.1.3.

The FO Eq. (2) with basal sliding at the bedrock Eq. (16) and stress-free boundary conditions Eq. (15) on the remaining boundaries were solved on the base $8 \mathrm{~km}$ resolution mesh and the four successively refined meshes. Model runs were performed in parallel on Titan ${ }^{5}$, a Cray XK6 operated by the Oak Ridge Leadership Computing Facility (OLCF). Note that the parallel decompositions employed in the runs were 2D only; all elements with the same $x$ and $y$ coordinates were on the same processor (convergence difficulties were encountered when splitting vertical columns in the mesh across processors). A parallel decomposition for 16 cores is illustrated in Fig. 10.

Tables 4 and 5 report the relative errors in the computed solution for each mesh resolution considered with uniform and graded vertical mesh spacings (respectively). The convergence metric employed was the continuous $L^{2}$ norm. The relative error in each solution was calculated according to the following formula:

$\mathcal{E}_{\mathrm{rel}}^{\mathrm{cont}} \equiv \sqrt{\frac{\int_{\Omega}\left\|\boldsymbol{u}_{n}-\boldsymbol{u}_{\mathrm{ref}}\right\|_{2}^{2} d \Omega}{\int_{\Omega}\left\|\boldsymbol{u}_{\mathrm{ref}}\right\|_{2}^{2} d \Omega}}$

In Eq. (46), $\|\cdot\|_{2}$ denotes the $L^{2}$ norm, $\boldsymbol{u}_{n}$ denotes the computed solution and $\boldsymbol{u}_{\text {ref }}$ denotes the reference solution, which here we take as the solution computed for the finest resolution mesh, the $500 \mathrm{~m}$ mesh with 80 vertical layers and graded vertical spacing (for this quasi-realistic problem, there is no exact solution available in closed analytic form). This finest mesh had 1.12 billion dofs. The integrals in Eq. (46) were calculated exactly using a sufficiently accurate numerical quadrature rule.

Below, we provide some discussion of the data summarized in Tables 4 and 5, as well as some conclusions drawn from these results.

\section{Mesh convergence}

Figure 11 shows the relative error Eq. (46) as a function of the horizontal mesh spacing $(8,4,2,1 \mathrm{~km})$ on a $\log$-log plot (blue line). The numerical values of the relative error $\mathcal{E}_{\text {rel }}$ plotted are the diagonal entries of Tables 4 and 5 (which were identical for the two tables). The asymptotic convergence rate (the slope of the blue line in Fig. 11 disregarding the coarsest mesh data point, as it is not in the region of asymptotic convergence) is 1.97 . This compares very well with the theoretical convergence rate of 2 , for the bilinear hexahedral elements considered in this norm (black-dashed line in Fig. 11).

\footnotetext{
${ }^{5}$ More information on Titan can be found at www.olcf.ornl.gov/ titan.
}

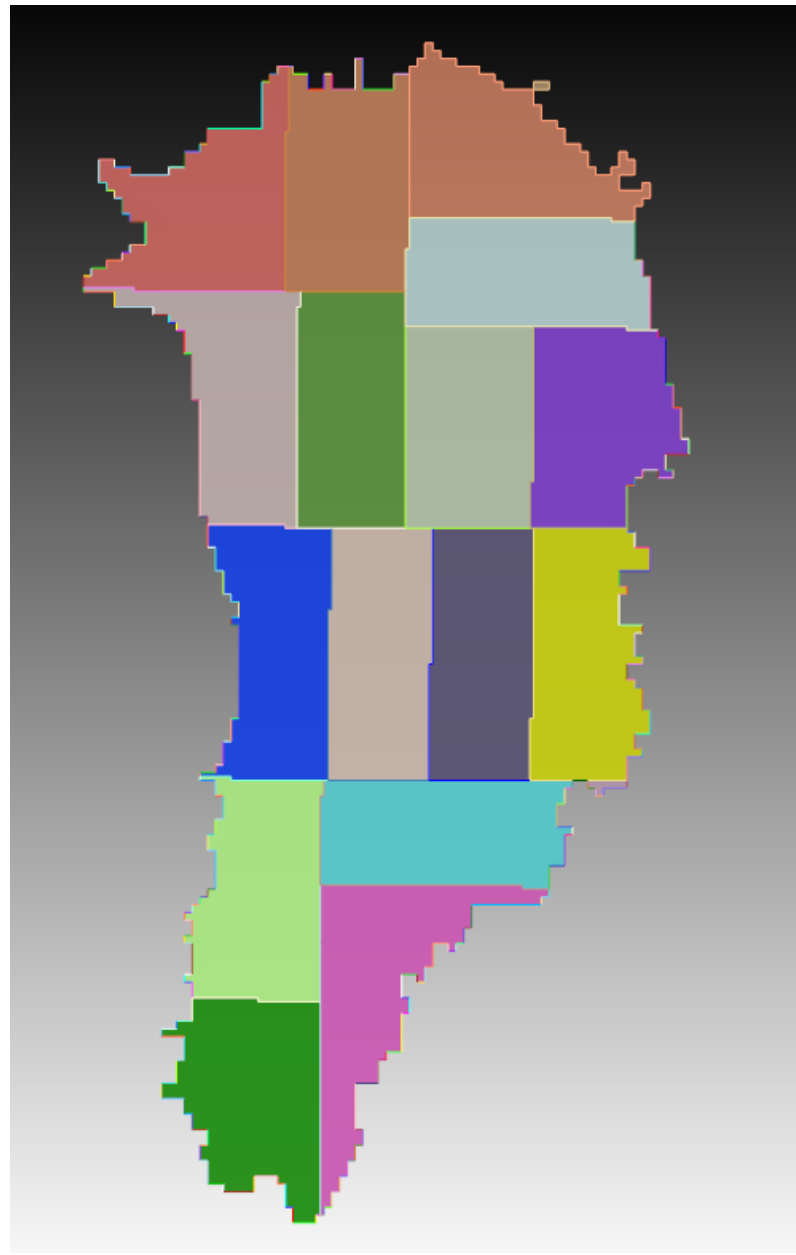

Figure 10. GIS domain decomposition for 16 core, parallel run, with different colors representing portions of the domain owned by different cores.

\section{Effect of partitioning on mesh convergence}

As noted in the discussion of the full 3-D mesh convergence study described in Sect. 6, our study revealed that 2-D parallel decompositions of the meshes (i.e., decompositions in which all elements with the same $x$ and $y$ coordinates were on the same processor, as shown in Fig. 10) led to out-of-thebox convergence of our linear and nonlinear solves. In contrast, convergence difficulties were encountered when splitting vertical columns in the mesh across processors. The 2 $\mathrm{D}$ parallel decomposition is therefore recommended over a full 3-D parallel decomposition, especially for problems on meshes with a finer vertical resolution.

\section{Uniform vs. graded vertical spacing}

The reader may observe in comparing Tables 4 and 5 that there is no significant difference between the errors in the solutions on the meshes with a uniform vertical resolution and 
Table 4. Relative errors for the GIS mesh convergence study with uniform vertical spacing.

\begin{tabular}{|c|c|c|c|c|c|c|}
\hline $\begin{array}{l}\text { Horizontal } \\
\text { resolution }\end{array}$ & $\begin{array}{l}\text { Vertical } \\
\text { layers }\end{array}$ & 5 & 10 & 20 & 40 & 80 \\
\hline $8 \mathrm{~km}$ & & $2.0 \times 10^{-1}$ & & & & \\
\hline $4 \mathrm{~km}$ & & $9.0 \times 10^{-2}$ & $7.8 \times 10^{-2}$ & & & \\
\hline $2 \mathrm{~km}$ & & $4.6 \times 10^{-2}$ & $2.4 \times 10^{-2}$ & $2.3 \times 10^{-2}$ & & \\
\hline $1 \mathrm{~km}$ & & $3.8 \times 10^{-2}$ & $8.9 \times 10^{-3}$ & $5.5 \times 10^{-3}$ & $5.1 \times 10^{-3}$ & \\
\hline $500 \mathrm{~m}$ & & $3.7 \times 10^{-2}$ & $6.7 \times 10^{-3}$ & $1.7 \times 10^{-3}$ & $3.9 \times 10^{-4}$ & $8.1 \times 10^{-5}$ \\
\hline
\end{tabular}

Table 5. Relative errors for the GIS mesh convergence study with graded vertical spacing.

\begin{tabular}{|c|c|c|c|c|c|c|}
\hline $\begin{array}{l}\text { Horizontal } \\
\text { resolution }\end{array}$ & $\begin{array}{l}\text { Vertical } \\
\text { layers }\end{array}$ & 5 & 10 & 20 & 40 & 80 \\
\hline $8 \mathrm{~km}$ & & $2.0 \times 10^{-1}$ & & & & \\
\hline $4 \mathrm{~km}$ & & $8.3 \times 10^{-2}$ & $7.8 \times 10^{-2}$ & & & \\
\hline $2 \mathrm{~km}$ & & $3.3 \times 10^{-2}$ & $2.4 \times 10^{-2}$ & $2.3 \times 10^{-2}$ & & \\
\hline $1 \mathrm{~km}$ & & $2.2 \times 10^{-2}$ & $7.3 \times 10^{-3}$ & $5.3 \times 10^{-3}$ & $5.1 \times 10^{-3}$ & \\
\hline $500 \mathrm{~m}$ & & $2.1 \times 10^{-2}$ & $4.7 \times 10^{-3}$ & $1.2 \times 10^{-3}$ & $2.6 \times 10^{-4}$ & - \\
\hline
\end{tabular}

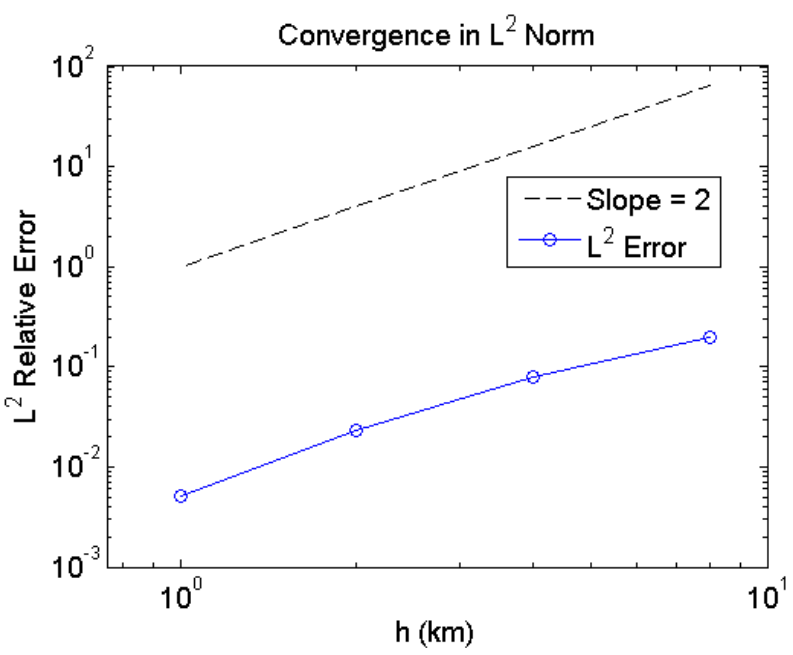

Figure 11. Convergence in the continuous $L^{2}$ norm Eq. (46) for the realistic GIS problem with full 3-D refinement.

those on meshes with a graded vertical resolution. Nonetheless, there is some value (at no additional computational cost) in using a graded mesh over a uniform mesh for some mesh resolutions.

\section{Practical recommendations on mesh resolution}

The data in Tables 4 and 5 suggest that, depending on the selected mesh resolution, there can be more value in refin- ing vertically than horizontally. For example, for the uniform mesh spacing (Table 4), the solution on a $2 \mathrm{~km}$ resolution mesh with 10 vertical layers is more accurate than that on a $1 \mathrm{~km}$ resolution mesh with 5 vertical layers. Similarly, the solution on a $1 \mathrm{~km}$ resolution mesh with 20 vertical layers is more accurate than that on a $500 \mathrm{~m}$ resolution mesh with 10 vertical layers. For the graded mesh spacing scenario (Table 5), a solution refined two times in $z$ is comparable in accuracy to the solution refined two times in the horizontal direction; however, the former problem is smaller, as refining two times vertically leads to a doubling of the number of dofs, whereas refining two times horizontally leads to a quadrupling of the number of dofs.

Although there can be value in refining vertically, this is true only up to some level of refinement. For each horizontal resolution in Tables 4 and 5 except the finest, the errors plateau beyond a certain vertical resolution. The data in the last row of the tables should be considered very cautiously, as here we are using the same horizontal resolution as the reference solution.

The results in Tables 4 and 5 can be used by readers to determine the horizontal and vertical mesh resolution required to attain a desired convergence rate. We note that the errors are for a controlled study, where the ice geometries are not changing with the horizontal resolution and the fields are smoother than in reality. When both the mesh resolution and the geometric data resolution increase simultaneously, hori- 
zontal refinement will likely be more important than Tables 4 and 5 suggest.

\subsection{Code performance and scalability}

Having demonstrated the numerical convergence of our code on a realistic, large-scale ice sheet problem, we now study the code's robustness, performance and scalability.

\subsubsection{Robustness}

In Sect. 3.1.1, we described our approach for improving the robustness of the nonlinear solver using a homotopy continuation of the regularization parameter (denoted by $\gamma$ ) appearing in the effective viscosity law expression Eq. (22). Here, we perform a numerical study of the relative robustness of Newton's method with and without the use of this continuation procedure on a realistic, $5 \mathrm{~km}$ resolution Greenland ice sheet problem. Three approaches are considered:

(a) full Newton with no homotopy continuation.

(b) Newton with backtracking but no homotopy continuation.

(c) full Newton with homotopy continuation.

For all three methods, a uniform velocity field is specified as the initial guess for Newton's method. To prevent the effective viscosity Eq. (8) from evaluating to "not-a-number" for this initial guess, we replace $\mu$ by $\mu_{\gamma}$ in Eq. (2), where $\mu_{\gamma}$ is given by Eq. (22) and $\gamma=10^{-10}$ for the first two approaches. The third approach implements Algorithm 1, in which we use a natural continuation algorithm to reach $\gamma$ $=10^{-10}$ starting with $\alpha_{0}=0.1$.

Figure 12 illustrates the performance of Newton's method for the three approaches considered by plotting the norm of the residual as a function of the total number of Newton iterations. The reader can observe that full Newton with no homotopy continuation diverges. If backtracking is employed, the algorithm converges to a tolerance of $10^{-4}$ in 43 nonlinear iterations. With the use of homotopy continuation, the number of nonlinear iterations is cut almost in half, to 24 nonlinear iterations. The natural continuation method leads to four homotopy steps.

It is well known that, for Newton's method to converge to the root of a nonlinear function (i.e., the solution to the discrete counterpart of Eq. 21), it must start with an initial guess that is reasonably close to the sought-after solution. The proposed homotopy continuation method is particularly useful in the case when no "good" initial guess is available for Newton's method, in which case the nonlinear solver may fail to converge (see Sect. 3.1.1 and Algorithm 1). Homotopy continuation may not be needed for robust convergence in the case that a "good" initial guess is available (e.g., from observations or from a previously converged model time step).

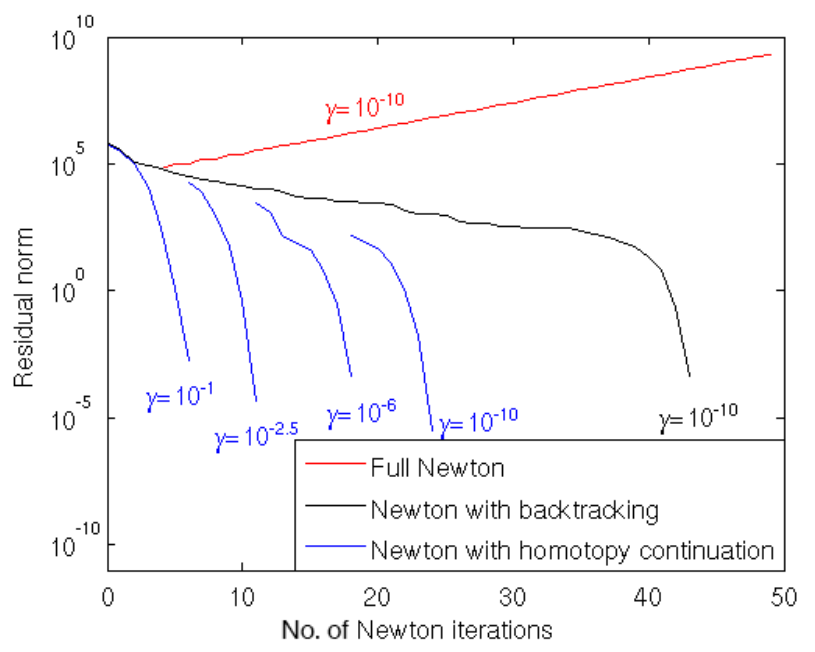

Figure 12. The robustness of Newton's method nonlinear solves with homotopy continuation.

Table 6. Meshes used in the GIS controlled weak scalability study.

\begin{tabular}{lrrr}
\hline $\begin{array}{l}\text { Horizontal } \\
\text { resolution }\end{array}$ & $\begin{array}{r}\text { No. of vertical } \\
\text { layers }\end{array}$ & No. of dofs & No. of cores \\
\hline $8 \mathrm{~km}$ & 5 & $3.34 \mathrm{~K}$ & 4 \\
$4 \mathrm{~km}$ & 10 & $2.43 \mathrm{M}$ & 32 \\
$2 \mathrm{~km}$ & 20 & $18.4 \mathrm{M}$ & 256 \\
$1 \mathrm{~km}$ & 40 & $143 \mathrm{M}$ & 2048 \\
$500 \mathrm{~m}$ & 80 & $1.12 \mathrm{~B}$ & 16384 \\
\hline
\end{tabular}

\subsubsection{Controlled weak scalability study on successively refined meshes with coarse mesh data}

First, we report results for a controlled weak scalability study. For this experiment, the $8 \mathrm{~km}$ GIS mesh with 5 vertical layers described in Sect. 6 was scaled up to a $500 \mathrm{~m}$ GIS mesh with 80 vertical layers using the uniform 3-D mesh refinement discussed earlier. A total of five meshes were generated, as summarized in Table 6. The term "controlled" refers to the fact that the resolution of the data describing the ice sheet geometry used for initial conditions was held fixed for all the grids considered and equal to the polygonal boundary determined by the coarsest $8 \mathrm{~km}$ mesh. Moreover, topography, surface height, basal friction and temperature data have been smoothed and then interpolated as described in Sect. 6. Each resolution problem was run in parallel on the Hopper $^{6}$ Cray XE6 supercomputer at the National Energy Research Scientific Computing (NERSC) Center. The number of cores for each run (third column of Table 6) was calculated so that, for each size problem, each core had approximately the same number of dofs $(\approx 70-80 \mathrm{~K}$ dofs per core). For a detailed discussion of the numerical methods

\footnotetext{
${ }^{6}$ More information on the Hopper machine can be found here: http://www.nersc.gov/users/computational-systems/hopper.
} 


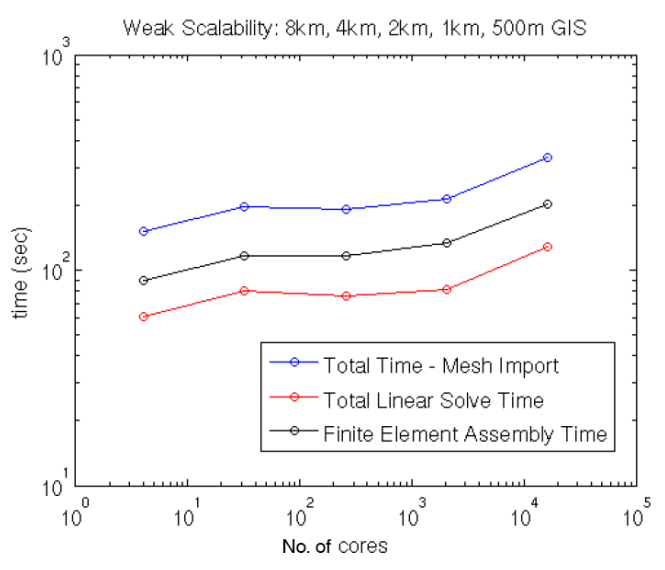

(a)

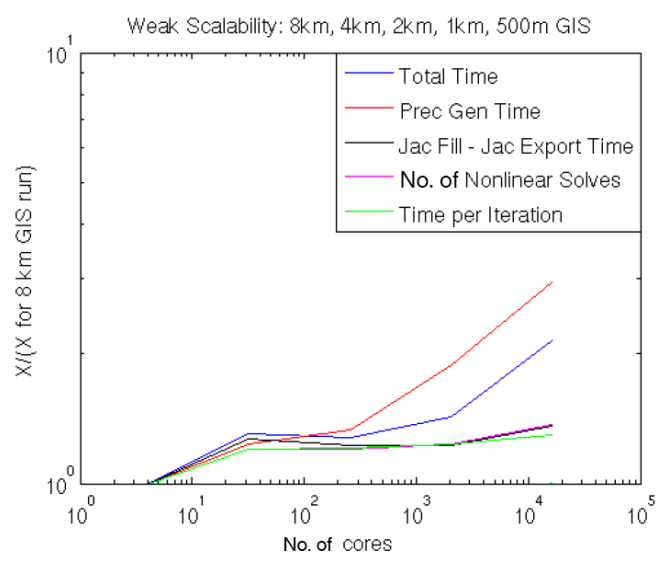

(b)

Figure 13. Controlled, weak scalability study on Hopper: (a) total linear solve, finite element assembly, and total run times in seconds, and (b) additional timing information $(X=$ time or number of iterations).

employed, the reader is referred to Sect. 3. In particular, recall that the linear solver employed is based on the preconditioned CG iterative method. The preconditioner employed is the algebraic multilevel preconditioner based on the idea of semi-coarsening that was described in Sect. 3.1.2. This preconditioner is available through the $M L$ package of Trilinos (Heroux et al., 2005).

Figure 13a reports the total linear solver time, the finite element (FE) assembly time and the total time (in seconds) for each resolution problem considered, as a function of the number of cores. Figure $13 \mathrm{~b}$ shows more detailed timing information, namely

- the normalized preconditioner generation time ("Prec Gen Time");

- the normalized Jacobian fill time, not including the Jacobian export time ${ }^{7}$ ("Jac Fill - Jac Export Time");

- the normalized number of nonlinear solves ("No. of Nonlin Solves");

- the normalized average number of linear iterations ("Avg no. of Lin Iter"); and

- the normalized total time not including I/O ("Total Time IO").

The run times and iteration counts have been normalized by the run time and iteration count (respectively) for the smallest run ( $8 \mathrm{~km}$ GIS with five vertical layers, run on four cores). Figure 13 reveals that the run times and iteration times scale well, albeit not perfectly, in a weak sense.

\footnotetext{
7"Jacobian export time" refers to the time required to transfer ("export") data from an element-based decomposition, which can be formed with no communication, to a node-based decomposition, where rows of the matrix are uniquely owned by a single processor.
}

\subsubsection{Strong scalability for realistic Greenland initial conditions on a variable-resolution mesh}

For the performance study described in the previous paragraph, the data have been smoothed and the lateral boundary was determined by the coarsest ( $8 \mathrm{~km}$ resolution) mesh. We now perform a scalability study for the GIS by directly interpolating the original data sets into the mesh considered. This results in better resolved topography, basal friction and temperature fields in regions of the domain with higher resolution. As before, the surface topography and temperature fields are from Bamber et al. (2013) and were generated as a part of the Ice2Sea project (Ice2sea, 2014); the optimized basal friction coefficient $(\beta)$ field is from Perego et al. (2014).

We consider a tetrahedral mesh with a variable resolution of between $1 \mathrm{~km}$ and $7 \mathrm{~km}$ and with approximately 14.4 million elements, leading to approximately 5.5 million dofs (Fig. 14a). The mesh was created by first meshing the base of the GIS using the 2-D meshing software Triangle (Shewchuk, 1996). The 2-D mesh generated using Triangle was a nonuniform Delaunay triangulation in which the areas of the triangles were constrained to be roughly proportional to the norm of the gradient of the surface velocity data. This yields meshes with better resolutions in places where the solution has larger variations. The 2-D mesh is then extruded in the $z$ direction as prisms and each prism is divided into three tetrahedra (Fig. 14b).

First, we verify that velocities computed on the $1-7 \mathrm{~km}$ variable resolution tetrahedral mesh, shown in Fig. 15a, agree with observations to within expectations. The reader can observe that there is generally good agreement between the modeled velocities and those from the target field observations, shown in Fig. 15b (from Joughin et al., 2010). Differences between the modeled and observed velocities occur as a result of the objective function used during model opti- 


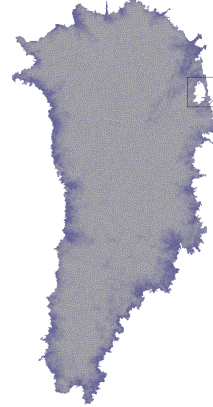

(a)

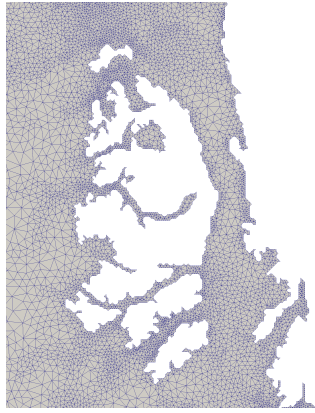

(b)

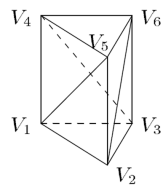

(c)
Figure 14. (a) Variable-resolution 1-7 km GIS mesh, (b) close-up of the variable-resolution 1-7 km GIS mesh (boxed region in a), and (c) subdivision of the hexahedral finite element into three tetrahedra.

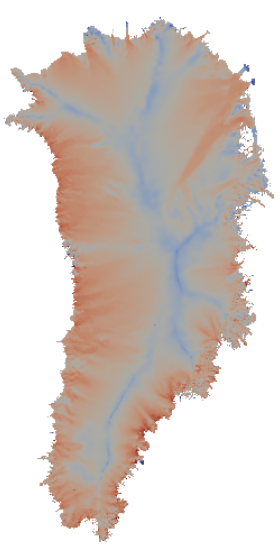

(a)

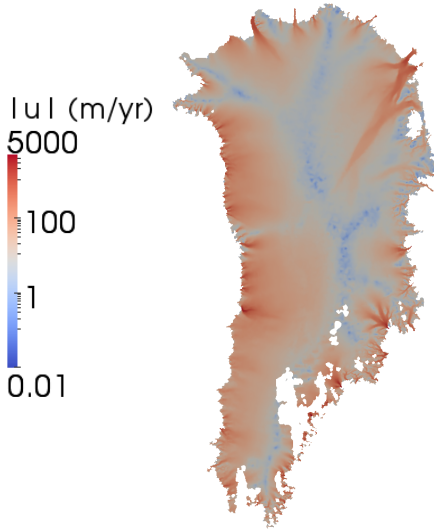

(b)
Figure 15. Solution magnitude $|\boldsymbol{u}|$ in meters per year: (a) $A l$ bany/FELIX solution (surface speed) on the variable resolution (1$7 \mathrm{~km}$ ) tetrahedral mesh, and (b) observed surface speeds (from Joughin et al. (2010)).

mization, which takes into account factors other than just the velocity mismatch ${ }^{8}$.

Next, a strong scaling study on the $1-7 \mathrm{~km}$ variable resolution GIS problem is performed. The problem is run on different numbers of cores on Hopper, from 64 to 512. The total solve, linear solve and finite element assembly times for each of the runs are reported (in seconds) in Table 7. The speed-up relative to the smallest (64 core) run is plotted as a function of the number of cores in Fig. 16. Good strong scalability is obtained: a 3.75 times speed-up is observed with 4 times the number of cores (up to and including 256

\footnotetext{
${ }^{8}$ The optimization procedure, discussed in more detail in Perego et al. (2014), minimizes the difference between modeled and observed velocities and between the modeled flux divergence and a target surface mass balance field. The latter constraint, which is introduced so that the optimized model is in quasi-steady state with climate model forcing, results in the small differences between the modeled and observed velocities observed in Fig. 15.
}

Table 7. Total, linear solve and finite element assembly times (s) for variable resolution $1-7 \mathrm{~km}$ resolution GIS problems as a function of the number of cores of Hopper.

\begin{tabular}{llll}
\hline No. of cores & $\begin{array}{l}\text { Total } \\
\text { solve time }\end{array}$ & $\begin{array}{l}\text { Linear } \\
\text { solve time }\end{array}$ & $\begin{array}{l}\text { Finite element } \\
\text { assembly time }\end{array}$ \\
\hline 64 & 268.1 & 119.9 & 148.3 \\
128 & 139.9 & 63.12 & 76.78 \\
256 & 78.41 & 37.92 & 40.49 \\
512 & 56.83 & 33.81 & 23.02 \\
\hline
\end{tabular}

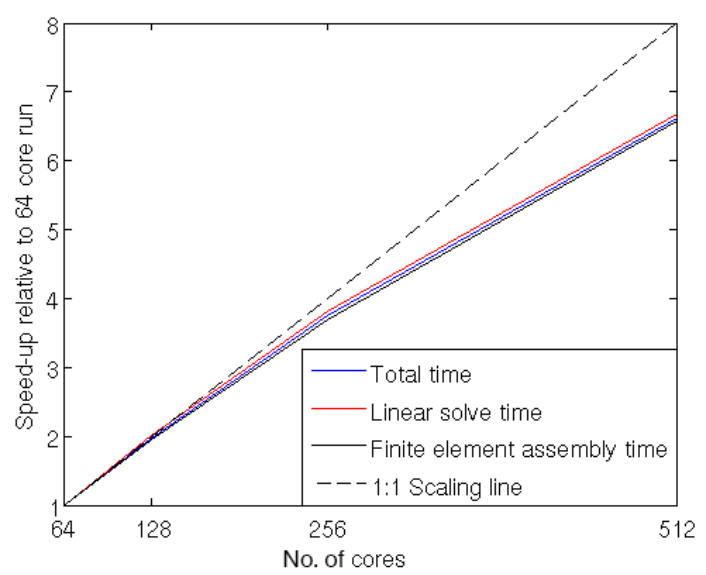

Figure 16. Strong scalability for the $1-7 \mathrm{~km}$ resolution GIS problem: speed-up relative to the 64 -core run.

cores), and a 6.64 times speed-up is observed with 8 times the number of cores (up to and including 512 cores). In these results, the linear solver employed was the preconditioned CG iterative method, with the aforementioned algebraic multilevel preconditioner based on the idea of semi-coarsening (see Sect. 3.1.2).

\section{Conclusions}

In this paper, we have presented a new, parallel, finite element solver for the first-order accurate, nonlinear Stokes ice sheet model. This solver, Albany/FELIX, has been written using a component-based approach to building application codes. The components comprising the code are modular Trilinos libraries, which are put together using abstract interfaces and template-based generic programming. Several verifications of the code's accuracy and convergence are carried out. First, a mesh convergence study is performed on several new methods of manufactured solution test cases derived for simplified 2-D versions of the first-order Stokes equations. All finite elements tested exhibit their theoretical rate of convergence. Next, code-to-code comparisons are made on several canonical ice sheet benchmarks between the $\mathrm{Al}$ bany/FELIX code and the finite element solver of Perego et 
al. (2012). The solutions are shown to agree to within machine precision. As a final verification, a mesh convergence study on a realistic Greenland geometry is performed. The purpose of this test is two-fold: (1) to demonstrate that the solution converges at the theoretical rate with mesh refinement, and (2) to determine how many vertical layers are required to accurately resolve the solution with a fixed $x-y$ resolution, when using (low-order) trilinear finite elements. It is found that the parallel decomposition of a mesh has some effect on the linear and nonlinear solver convergence: better performance is observed on the finer meshes if a horizontal decomposition (i.e., a decomposition in which all nodes with the same $x$ and $y$ coordinates are on the same processor) is employed for parallel runs. Further performance studies reveal that a robust nonlinear solver is obtained through the use of homotopy continuation with respect to a regularization parameter in the effective viscosity in the governing equations, and that good weak scalability can be achieved by preconditioning the iterative linear solver using an algebraic multilevel preconditioner constructed based on the idea of semi-coarsening.
Finally, we note that the ultimate purpose for developing Albany/FELIX is to integrate it into more complete land ice modeling frameworks so that it can be used for prognostic simulations, both in standalone mode and as a coupled component of ESMs. In addition to the conservation of linear momentum being solved by Albany/FELIX, a complete prognostic land ice model must also solve discretized PDEs for the conservation of mass and energy, in addition to treating other physical processes such as lithospheric heat exchange and isostatic bedrock adjustment. To enable prognostic runs, we have written interfaces for coupling Albany/FELIX to two larger land ice modeling frameworks, which discretize and solve the equations for mass and energy conservation: the Community Ice Sheet Model version 2.0 (CISM2) (Price et al., 2014) and the Model for Prediction Across Scales Land Ice (MPAS-LI) (Hoffman, 2013). We refer to the resulting complete land ice models as CISM-Albany and MPASAlbany respectively. Prognostic runs using these dycores are iterative in nature, with a diagnostic solve for the velocity field occurring in Albany/FELIX, followed by solutions for the geometry and temperature evolution occurring in CISM2 or MPAS-LI. Further discussion of CISM-Albany and MPASAlbany and ongoing work involving their coupling to ESMs will be deferred to subsequent papers. Similarly, these combined codes will be publicly released at a later point in time. 


\section{Appendix A: Nonlinear Stokes model for glaciers and ice sheets}

The model considered here, referred to as the first-order (FO) Stokes approximation, or the Blatter-Pattyn model (Blatter, 1995; Pattyn, 2003), is an approximation of the nonlinear Stokes model for glacier and ice sheet flow. In general, glaciers and ice sheets are modeled as an incompressible fluid in a low Reynolds number flow with a power-law viscous rheology, as described by the Stokes flow equations. The equations are quasi-static, as the inertial and advective terms can be neglected due to the slow movement of the ice.

Let $\sigma$ denote the Cauchy stress tensor, given by

$\boldsymbol{\sigma}=2 \mu \dot{\boldsymbol{\epsilon}}-p \mathbf{I} \in \mathbb{R}^{3 \times 3}$,

where $\mu$ denotes the "effective" ice viscosity, $p$ the ice pressure, I the identity tensor, and $\dot{\boldsymbol{\epsilon}}$ the strain-rate tensor:

$\dot{\epsilon}_{i j}=\frac{1}{2}\left(\frac{\partial u_{i}}{\partial x_{j}}+\frac{\partial u_{j}}{\partial x_{i}}\right)$,

for $i, j \in\{1,2,3\}$. The effective viscosity is given by Glen's law (Nye, 1957; Cuffey and Paterson, 2010):

$\mu=\frac{1}{2} A^{-\frac{1}{n}} \dot{\epsilon}_{\mathrm{e}}^{\left(\frac{1}{n}-1\right)}$,

where

$\dot{\epsilon}_{\mathrm{e}}=\sqrt{\frac{1}{2} \sum_{i j} \dot{\epsilon}_{i j}^{2}}$ denotes the effective strain rate, given by the second invariant of the strain-rate tensor. $A$ denotes the flow rate factor (which is strongly dependent on the ice temperature), and $n$ denotes the power law exponent (generally taken equal to 3 ). The nonlinear Stokes equations for glacier and ice sheet flow can then be written as follows:

$\left\{\begin{aligned}-\nabla \cdot \boldsymbol{\sigma} & =\rho \mathbf{g} \\ \nabla \cdot \boldsymbol{u} & =0 .\end{aligned}\right.$

Here, $\rho$ denotes the ice density, and $\mathbf{g}$ the gravitational acceleration vector, i.e., $\mathbf{g}^{T}=(0,0,-g)$, with $g$ denoting the gravitational acceleration. The values of the parameters that appear in the expressions above are given in Table 1. A stressfree boundary condition is prescribed on the upper surface:

$\boldsymbol{\sigma n}=\mathbf{0}$, on $\Gamma_{s}$.

On the lower surface, the relevant boundary condition is the no-slip or basal sliding boundary condition

$\left\{\begin{array}{l}\boldsymbol{u}=\mathbf{0}, \text { on } \Gamma_{0}, \\ \boldsymbol{u} \cdot \boldsymbol{n}=0 \text { and }(\boldsymbol{\sigma} \boldsymbol{n}+\beta \boldsymbol{u})_{\|}=\mathbf{0}, \text { on } \Gamma_{\beta},\end{array}\right.$

assuming $\Gamma_{b}=\Gamma_{0} \cup \Gamma_{\beta}$ with $\Gamma_{0} \cap \Gamma_{\beta}=\varnothing$, where $\beta \equiv$ $\beta(x, y) \geq 0$. The operator $(\cdot)_{\|}$in Eq. (53) performs the tangential projection onto a given surface. 


\section{Code Availability}

The Albany framework is an open-source development project available for download on GitHub (http://gahansen. github.io/Albany). Albany, currently on its 2.0 release, is released under a publicly available designation with a threeterm BSD license.

The Albany framework was written using many libraries available through Trilinos, also publicly available (http:// trilinos.org). At the time this journal article was written, Trilinos was on its 11.12 release. The multigrid algorithm presented in this paper (Sect. 3.1.2) is implemented within the $M L$ package of Trilinos and is available for use with Trilinos 11.12 or later.

The Albany/FELIX solver described in this paper is not publicly available at the present time. A public release of the code as part of Albany is planned for 2015. The CISM and MPAS ice sheet models with supported interfaces to $\mathrm{Al}$ bany/FELIX will also be made publicly available at a later point in time.

Acknowledgements. Support for all authors was provided through the Scientific Discovery through Advanced Computing (SciDAC) program funded by the U.S. Department of Energy (DOE), Office of Science, Advanced Scientific Computing Research and Biological and Environmental Research. This research used resources of the National Energy Research Scientific Computing Center (NERSC; supported by the Office of Science of the U.S. Department of Energy under contract DE-AC02-05CH11231) and the Oak Ridge Leadership Computing Facility (OLCF; supported by the DOE Office of Science under contracts DE-AC02-05CH11231 and DE-AC05-00OR22725). The authors thank M. Norman of Oak Ridge National Laboratory for the generation of the Greenland geometry data sets, J. Johnson (and students) of the University of Montana for initial development of the ISMIP-HOM plotting scripts, and M. Hoffman and B. Lipscomb at Los Alamos National Laboratory for useful discussions that led to some of the ideas and results presented in this paper.

Edited by: N. Kirchner

\section{References}

Adams, B., Bauman, L., Bohnhoff, W., Dalbey, K., Ebeida, M., Eddy, J., Eldred, M., Hough, P., Hu, K., Jakeman, J., Swiler, L., and Vigil, D.: DAKOTA, A Multilevel Parallel Object-Oriented Framework for Design Optimization, Parameter Estimation, Uncertainty Quantification, and Sensitivity Analysis: Version 5.4 User's Manual, Sandia Technical Report SAND2010-2183, December 2009, updated April 2013.

Allgower, E. and Georg, K.: Introduction to Numerical Continuation Methods, SIAM Classics in Applied Mathematics, 45, 2003.

Balay, S., Buschelman, K., Eijkhout, V., Gropp, W., Kaushik, D., Knepley, M., McInnes, L., Smith, B., and Zhang, H.: PETSc Users Manual, Technical Report ANL-95/11, Revision 3.0.0, Argonne National Laboratory, Lemont, IL, 2008.
Bamber, J. L., Griggs, J. A., Hurkmans, R. T. W. L., Dowdeswell, J. A., Gogineni, S. P., Howat, I., Mouginot, J., Paden, J., Palmer, S., Rignot, E., and Steinhage, D.: A new bed elevation dataset for Greenland, The Cryosphere, 7, 499-510, doi:10.5194/tc-7499-2013, 2013.

Bavier, E., Hoemmen, M., Rajamanickam, S., and Thornquist, H.: Amesos2 and Belos: Direct and iterative solvers for large sparse linear systems, Sci. Program., 20, 241-255, 2012.

Blatter, H.: Velocity and stress fields in grounded glaciers: a simple algorithm for including deviatoric stress gradients, J. Glaciol., 41, 333-344, 1995.

Bochev, P., Edwards, C., Kirby, R., Peterson, K., and Ridzal, D.: Solving PDEs with Intrepid, Sci. Program., 20, 151-180, 2012.

Brinkerhoff, D. J. and Johnson, J. V.: Data assimilation and prognostic whole ice sheet modelling with the variationally derived, higher order, open source, and fully parallel ice sheet model VarGlaS, The Cryosphere, 7, 1161-1184, doi:10.5194/tc-7-11612013, 2013.

Brown, P., Falgout, R., and Jones, J.: Semi-coarsening Multigrid on Distributed Memory Machines, J. Sci. Comput., 21, 1823-1834, 2000.

Brown, J., Smith, B., and Ahmadia, A.: Achieving textbook multigrid efficiency for hydrostatic ice sheet flow, J. Sci. Comput., 35, B359-B375, 2013.

Bueler, E. and Brown, J.: Shallow shelf approximation as a "sliding law" in a thermomechanically coupled ice sheet model, J. Geophys. Res., 114, 1-21, doi:10.1029/2008JF001179, 2009.

Bueler, E., Brown, J., and Lingle, C.: Exact solutions to the thermomechanically coupled shallow-ice approximation: effective tools for verification, J. Glaciol., 53, 499-516, 2007.

Chen, Q., Gunzburger, M., and Perego, M.: Well-Posedness Results for a Nonlinear Stokes Problem Arising in Glaciology, J. Math. Anal., 45, 2710-2733, 2013.

Cornford, S., Martin, D., Graves, D., Ranken, D., Le Brocq, A., Gladstone, R., Payne, A., Antony, J., Ng, E., and Lipscomb, W.: Adaptive mesh, finite volume modeling of marine ice sheets, J. Comput. Phys., 232, 529-549, doi:10.1016/j.jcp.2012.08.037, 2013.

Cuffey, K. and Paterson, W.: The physics of glaciers, 4th edition, Butterworth-Heinemann, Oxford, 2010.

Dendy, J. and Moulton, J.: Black box multigrid with coarsening by a factor of three, Numer. Linear. Algebr., 17, 577-598, 2010.

Dukowicz, J., Price, S., and Lipscomb, W.: Consistent approximations and boundary conditions for ice-sheet dynamics from a principle of least action, J. Glaciol., 56, 480-496, 2010.

Gagliardini, O., Zwinger, T., Gillet-Chaulet, F., Durand, G., Favier, L., de Fleurian, B., Greve, R., Malinen, M., Martín, C., Råback, P., Ruokolainen, J., Sacchettini, M., Schäfer, M., Seddik, H., and Thies, J.: Capabilities and performance of Elmer/Ice, a newgeneration ice sheet model, Geosci. Model Dev., 6, 1299-1318, doi:10.5194/gmd-6-1299-2013, 2013.

Gao, X., Nielsen, E., Muller, R., Young, R., Salinger, A., Bishop, N., Lilly, M., and Carroll, M.: Quantum computer aided design simulation and optimization of semiconductor quantum dots, J. Appl. Phys., 114, 1-19, 2013.

Gee, M., Siefert, C., Hu, J., Tuminaro, R., and Sala, M.: ML 5.0 smoothed aggregation user's guide, Sandia National Laboratories Report, SAND2006-2649, Sandia National Laboratories, Albuquerque, NM, 2007. 
Gillet-Chaulet, F., Gagliardini, O., Seddik, H., Nodet, M., Durand, G., Ritz, C., Zwinger, T., Greve, R., and Vaughan, D. G.: Greenland ice sheet contribution to sea-level rise from a new-generation ice-sheet model, The Cryosphere, 6, 1561-1576, doi:10.5194/tc-6-1561-2012, 2012.

Goldberg, D. N. and Heimbach, P.: Parameter and state estimation with a time-dependent adjoint marine ice sheet model, The Cryosphere, 7, 1659-1678, doi:10.5194/tc-7-1659-2013, 2013.

Goldberg, D. N. and Sergienko, O. V.: Data assimilation using a hybrid ice flow model, The Cryosphere, 5, 315-327, doi:10.5194/tc-5-315-2011, 2011.

Heroux, M., Bartlett, R., Howle, V., Hoekstra, R., Hu, J., Kolda, T., Lehoucq, R., Long, K., Pawlowski, R., Phipps, E., Salinger, A., Thornquist, H., Tuminaro, R., Willenbring, J., Williams, A., and Stanley, K.: An Overview of the Trilinos Project, Trans. Math. Softw., 31, 397-423, 2005.

Hoffman, M.: MPAS-Land Ice Model User's Guide Version: 3.0., available at: http://oceans11.lanl.gov/mpas_data/mpas_landice/ users_guide/release_3.0/mpas_landice_users_guide_3.0.pdf (last access: 15 March 2015), 2013.

Hughes, T.: The Finite Element Method: Linear Static and Dynamic Finite Element Analysis, Dover edition, 2000.

Hutter, K.: Theoretical glaciology: material science of ice and the mechanics of glaciers and ice sheets, Springer, 1983.

Ice2sea: British Antarctic Survey: ice2sea FP7 EU Project Homepage, available at: http://www.ice2sea.eu (last access: 15 March 2015), 2014.

IPCC: Solomon, S., Qin, D., Manning, M., Chen, Z., Marquis, M., Averyt, K., Tignor, M., and Miller, H.: Climate change 2007: The physical science basis, Contribution of Working Group I to the Fourth Assessment Report of the Intergovernmental Panel on Climate Change, Cambridge Univ Press, Cambridge, UK, 2007.

Isaac, T., Stadler, G., and Ghattas, O.: Solution of nonlinear Stokes equations discretized by high-order finite elements on nonconforming and anisotropic meshes, with application to ice sheet dynamics, submitted, available at: http://arxiv.org/abs/1406.6573, 2014.

Joughin, I., Smith, B., Howat, I., Scambos, T., and Moon, T.: Greenland flow variability from ice-sheet-wide velocity mapping, J. Glaciol., 56, 415-430, 2010.

Jouvet, G. and Graser, C.: An adaptive Newton multigrid method for a model of marine ice sheets, J. Computat. Phys., 252, 419-437, doi:10.1016/j.jcp.2013.06.032, 2013.

Khazendar, A., Rignot, E., and Larour, E.: Roles of marine ice, rheology, and fracture in the flow and stability of the Brunt/Stancomb-Wills Ice Shelf, J. Geophys. Res., 114, 1-9, doi:10.1029/2008JF001124, 2009.

Larour, E., Seroussi, H., Morlighem, M., and Rignot, E.: Continental scale, high order, high spatial resolution, ice sheet modeling using the Ice Sheet System Model (ISSM), J. Geophys. Res., 117, 1-20, doi:10.1029/2011JF002140, 2012.

Lemieux, J.-F., Price, S., Evans, K., Knoll, D., Salinger, A., Holland, D., and Payne, A.: Implementation of the Jacobian-free Newton-Krylov method for solving first-order ice sheet momentum balance, J. Comput. Phys., 230, 6531-6545, 2011.

Leng, W., Ju, L., Gunzburger, M., Price, S., and Ringler, T.: A Parallel Higher-Order Accurate Finite Element Nonlinear Stokes Ice Sheet Model and Benchmark Experiments, J. Geophys. Res., 117, 1-24, 2012.
Leng, W., Ju, L., Gunzburger, M., and Price, S.: Manufactured solutions and the verification of three-dimensional Stokes ice-sheet models, The Cryosphere, 7, 19-29, doi:10.5194/tc-7-19-2013, 2013.

Leng, W., Ju, L., Gunzburger, M., and Price, S.: A Parallel Computational Model for Three-Dimensional, Thermo-Mechanical Stokes Flow Simulations of Glaciers and Ice Sheets, Commun. Comput. Phys., in press, available at: http://www.global-sci.com/ galley/CiCP-272.pdf, 2015.

Lipscomb, W., Bindschadler, R., Price, S., Bueler, E., Johnson, J., and Holland, D.: A community ice sheet model for sea level prediction, Eos. Trans. AGU, 90, p. 23, 2008.

Lipscomb, W., Fyke, J., Vizcaino, M., Sacks, W., Wolfe, J., Vertenstein, M., Craig, A., Kluzek, E., and Lawrence, D.: Implementation and Initial Evaluation of the Glimmer Community Ice Sheet Model in the Community Earth System Model, J. Climate, 26, 7352-7371, doi:10.1175/JCLI-D-12-00557.1, 2013.

Little, C.: Toward a new generation of ice sheet models, Eos. Trans. AGU, 88 578-579, 2007.

MacAyeal, D., Rommelaere, V., Huybrechts, P., Hulbe, C., Datemann, J., and Ritz, C.: An ice-shelf model test based on the Ross Ice Shelf, Antarctica, Ann. Glaciol., 23, 46-51, 1996.

Morland, L.: Unconfined ice-shelf flow, in: Dynamics of the West Antarctic Ice Sheet Proc. Workshop held in Utrecht, May 6-8, 1985, edited by: van der Veen, C. and Oerlemans, J., 99-116, 1987.

Morlighem, M., Rignot, E., Seroussi, H., Larour, E., Ben Dhia, H., and Aubry, D.: Spatial patterns of basal drag inferred using control methods from a full-Stokes and simpler models for Pine Island Glacier, West Antarctica, Geophys. Res. Lett., 37, 1-6, doi:10.1029/2010GL043853, 2010.

Nye, J.: The Distribution of Stress and Velocity in Glaciers and IceSheets, P. Roy. Soc. A-Math. Phy., 239, 113-133, 1957.

Pattyn, F.: Transient glacier response with a higher-order numerical ice-flow model, J. Glaciol., 48, 467-477, 2002.

Pattyn, F.: A new three-dimensional higher-order thermomechanical ice-sheet model: basic sensitivity, ice stream development, and ice flow across subglacial lakes, J. Geophys. Res., 108, 115, 2003.

Pattyn, F., Perichon, L., Aschwanden, A., Breuer, B., de Smedt, B., Gagliardini, O., Gudmundsson, G. H., Hindmarsh, R. C. A., Hubbard, A., Johnson, J. V., Kleiner, T., Konovalov, Y., Martin, C., Payne, A. J., Pollard, D., Price, S., Rückamp, M., Saito, F., Souček, O., Sugiyama, S., and Zwinger, T.: Benchmark experiments for higher-order and full-Stokes ice sheet models (ISMIPHOM), The Cryosphere, 2, 95-108, doi:10.5194/tc-2-95-2008, 2008.

Pawlowski, R., Shadid, J., Simonis, J., and Walker, H.: Globalization techniques for Newton-Krylov methods and applications to the fully coupled solution of the Navier-Stokes equations, SIAM Rev., 48, 700-721, 2006.

Pawlowski, R., Phipps, E., and Salinger, A.: Automating embedded analysis capabilities and managing software complexity in multiphysics simulation, Part I: Template-based generic programming, Sci. Program., 20, 197-219, 2012.

Pawlowski, R., Phipps, E., Salinger, A., Owne, S., Siefert, C., and Staten, M.: Automating embedded analysis capabilities and managing software complexity in multiphysics simulation, Part II: 
Application to partial differential equations, Sci. Program., 20, 327-345, 2012.

Perego, M., Gunzburger, M., and Burkardt, J.: Parallel finiteelement implementation for higher-order ice-sheet models, J. Glaciol., 58, 76-88, 2012.

Perego, M., Price, S., and Stadler, G.: Optimal Initial Conditions for Coupling Ice Sheet Models to Earth System Models, J. Geophys. Res., 119, 1894-1917, doi:10.1002/2014JF003181, 2014.

Petra, N., Martin, J., Stadler, G., and Ghattas, O.: A computational framework for infinite-dimensional Bayesian inverse problems: Part II. Stochastic Newton MCMC with application to ice sheet inverse problems, J. Sci. Comput., in press, available at: http: //arxiv.org/abs/1308.6221, 2015.

Phipps, E. and Pawlowski, R.: Efficient Expression Templates for Operator Overloading-based Automatic Differentiation, in: Recent Advances in Algorithmic Differentiation, edited by: Forth, S., Hovland, P., Phipps, E., Utke, J., and Walther, A., Springer, 2012.

Pollard, D. and Deconto, R.: Modelling West Antarctic ice sheet growth and collapse through the past five million years, Nature, 458, 329-332, doi:10.1038/nature07809, 2009.

Price, S., Lipscomb, W., Hoffman, M., Hagdorn, M., Rutt, I., Payne, A. J., and Hebeler, F.: CISM 2.0.0 Documentation, available at: http://oceans11.lanl.gov/trac/CISM/documentation.html (last access: 15 March 2015), 2014.

Price, S., Lipscomb, W., Hoffman, M., Hagdorn, M., Rutt, I., Payne, T., and Hebeler, F.: CISM 2.0.0 Documentation, available at: http://oceans11.lanl.gov/cism/data/cism_documentation_v.0. pdf, last access: 22 April 2015.

Rommelaere, V.: Ice Shelf Models Intercomparison: setup of the experiments, unpublished document, available at: http://homepages.vub.ac.be/ phuybrec/eismint/shelf-descr.pdf (last access: 15 March 2015), 1996.

Rutt, I., Hagdorn, M., Hulton, N., and Payne, A.: The Glimmer community ice sheet model, J. Geophys. Res., 114, 1-22, 2009.

Salinger, A., Bou-Rabee, N., Burroughs, E., Lehoucq, R., Pawlowski, R., Romero, L., and Wilkes, E.: LOCA 1.0: Theory and Implementation Manual, Sandia Labs Technical Report, SAND2002-0396, 2002.

Salinger, A., Burroughs, E., Pawlowski, R., Phipps, E., and Romero, L.: Bifurcation tracking algorithms and software for large scale applications, Int. J. Bifurcat. Chaos, 3, 1015-1032, 2005.

Salinger, A., Phipps, E., Hansen, G., Kalashnikova, I., Ostien, J., Sun, W., Chen, Q., Mota, A., Muller, R., Nielsen, E., and Gao, X.: Albany: A Component-Based Partial Differential Equation Code Built on Trilinos, Sandia Labs Technical Report, SAND20138430, 2013.
Schaffer, S.: A semi-coarsening multigrid method for elliptic partial differential equations with highly discontinuous and anisotropic coefficients, J. Sci. Comput., 20, 228-242, 1998.

Schoof, C.: Coulomb friction and other sliding laws in a higherorder glacier flow model, Math. Mod. Meth. Appl. S., 20, 157189, 2010.

Schoof, C. and Hindmarsh, R.: Thin-Film Flows with Wall Slip: An Asymptotic Analysis of Higher Order Glacier Flow Models, Q. J. Mech. Appl. Math., 63, 73-114, 2010.

Seroussi, H., Ben Dhia, H., Morlighem, M., Larour, E., Rignot, E., and Aubry, D.: Coupling ice flow models of varying orders of complexity with the Tiling method, J. Glaciol., 58, 776-786, doi:10.3189/2012JoG11J195, 2012.

Shannon, S., Payne, A., Bartholomew, I., van der Broeke, M., Edwards, T., Fettweis, X., Gagliardini, O., Gillet-Chaulet, F., Goelzer, H., Hoffman, M., Huybrechts, P., Mair, D., Nienow, P., Perego, M., Price, S., Smeets, P., Sole, A., van de Wal, R., and Zwinger, T.: Enhanced basal lubrication and the contribution of the Greenland ice sheet to future sea-level rise, P. Natl. Acad. Sci. USA, 110, 14156-14161, doi:10.1073/pnas.1212647110, 2013.

Shewchuk, J.: Triangle: Engineering a 2-D Quality Mesh Generator and Delaunay Triangulator, in: Applied Computational Geometry: Towards Geometric Engineering, 1148, Lecture Notes in Compute Science, 203-222, Springer-Verlag, 1996.

Strang, W. and Fix, G.: Analysis of the finite element method, Prentice-Hall series in automatic computation, 1973.

Sun, W., Ostien, J., and Salinger, A.: A stabilized assumed deformation gradient finite element formulation for strongly coupled poromechanical simulations at finite strain, Int. J. Numer. Anal. Met., 37, 2755-2788, 2013.

Tuminaro, R.: ML's Semi-Coarsening Feature, Addition to ML 5.0 Smoothed Aggregation User's Guide, Sandia National Laboratories Report, SAND2006-2649, Sandia National Laboratories, Albuquerque, NM, available at: http://www.trilinos.org/wordpress/ wp-content/uploads/2014/08/SemiCoarsen.pdf (last access: 15 March 2015), 2014.

Tuminaro, R., Kalashnikova, I., Perego, M., and Salinger, A.: A hybrid operator dependent multi-grid/algebraic multi-grid approach: Application to ice sheet modeling, in preparation, 2015.

van der Veen, C. and ISMASS (Ice Sheet Mass Balance and Sea Level) Working Group: Ice Sheet Mass Balance and Sea Level: A Science Plan, Scientific Committee on Antarctic Research (SCAR) Report No. 38, ISSN 1755-9030, 2010. 